\title{
Provenance of fluvial sandstones at the start of late Jurassic-Early Cretaceous rifting in the Cameros Basin (N. Spain)
}

\author{
Laura González-Acebrón $^{\mathrm{a}, *}$, José Arribas ${ }^{\mathrm{b}}$, Ramón Mas ${ }^{\mathrm{a}}$ \\ ${ }^{a}$ Dto. Estratigrafia, Facultad de Ciencias Geológicas (UCM)-Instituto de Geología Económica (CSIC), \\ C/ Jose Antonio Novais 2, 28040 Madrid, Spain \\ ${ }^{\mathrm{b}}$ Dto. Petrología y Geoquímica, Facultad de Ciencias Geológicas (UCM)-Instituto de Geología Económica (CSIC), \\ C/ Jose Antonio Novais 2, 28040 Madrid, Spain
}

\begin{abstract}
The Cameros Basin (Iberian Chain, Central Spain) developed during the latest Jurassic-Early Cretaceous in an extensional regime characterized by high rates of subsidence. Its sedimentary fill has been subdivided into eight depositional sequences (DS) mainly composed of continental sediments. DS 1 and DS 2 represent the first rifting stage (Tera Group, Tithonian). The purpose of this study is to characterize the Tera Group in the eastern part of the basin based on provenance criteria derived from fluvial sandstones. In this area of the basin, the Tera Group can be subdivided into three formations: the Ágreda Formation, the Magaña Formation and the Sierra de Matute Formation. These formations are composed of alluvial-fan deposits, meandering fluvial sediments and lacustrine-palustrine mudstones.

A quantitative petrographic study indicated the presence of three main petrofacies in the Tera Group. The close correlation between petrofacies and lithostratigraphic units indicates that sandstone composition is a powerful tool for deciphering the tectonic processes active during the initial rift stages of the Cameros Basin.

Petrofacies 1 is sedimentolithic (mean: $\mathrm{Qm}_{54} \mathrm{~F}_{3} \mathrm{Lt}_{43}$ ) and represents erosion of the Jurassic marine pre-rift substratum (mainly Kimmeridgian limestones) during deposition of the DS 1 alluvial fan deposits (Ágreda Fm.). Petrofacies 2 is quartzofeldspathic, and can be subdivided into Petrofacies $2 \mathrm{~A}$, with an average composition of $\mathrm{Qm}_{84} \mathrm{~F}_{15} \mathrm{Lt}_{1}$ and Petrofacies $2 \mathrm{~B}$, whose average composition is $\mathrm{Q}_{71} \mathrm{~F}_{23} \mathrm{Lt}_{6}$. Petrofacies 2 was generated by the erosion of low to medium-grade metamorphic terranes and plutonic source rocks. It characterizes the Magaña Fm. (DS 2). Petrofacies 3 is quartzolithic (mean: $\mathrm{Qm}_{67} \mathrm{~F}_{16} \mathrm{Lt}_{17}$ ), and is attributed to tectonic reactivation of the basin. This petrofacies characterizes the Sierra de Matute Fm. (DS 2). Thus, the provenance evolution of this basin is characterized by erosion of the pre-rift sedimentarv substratum, followed by unroofing of the basement, as recorded in other ancient and modern rifted basins.
\end{abstract}

Keywords: Sandstone provenance; Depositional sequences; Tithonian; Cameros Basin; Northern Spain

\section{Introduction}

Sandstone petrography is widely considered to be a powerful tool for tectonic reconstructions and for deter-

\footnotetext{
* Corresponding author. Tel.: +34 91 3944785; fax: +34 91394480 E-mail address: lgcebron@geo.ucm.es (L. González-Acebrón).
}

mining the origin of ancient terrigenous deposits (Blatt, 1967; Dickinson, 1970; Pettijohn et al., 1972). Many factors, such as source area lithology, relief, climate and diagenesis affect the final sandstone composition. Several authors have described a relationship between the detrital composition of sandstones and the tectonic setting (e.g. Ingersoll, 1978; Dickinson and Suczek, 1979; Dickinson et al., 1983; Dickinson, 1985; Valloni, 1985). 
The provenance of foreland-basin arenites has been extensively investigated (Schwab, 1986; DeCelles and Hertel, 1989; Criteri and Le Pera, 1994; Critelli, 1999). In this type of basin, large volumes of clastic sediments are produced by erosion of thrust belts, and sandstoneprovenance analysis provides valuable insights into the record of thrust movement. Relatively few studies, however, have examined detailed processes and responses within intra-plate rift basins (Zuffa et al., 1980; Evans, 1990; Garzanti et al., 2001; Arribas et al., 2003; Garzanti et al., 2003). Multi-phase rifting and tilted crustal blocks lead to erosion and sediment redistribution within the basin, such that detrital modes of syn-rift sandstones strongly vary in relation to their paleotectonic position in the basin. The first stage of rifting usually starts with erosion of the pre-rift sedimentary substratum, followed by unroofing of the basement. Thus, sandstone provenance studies are essential for reconstructing eroded sediments and the tectonic evolution of rift basins.

The principal aims of this paper are: (1) to describe and discuss petrologic data derived from fluvial sandstones (Tera Group) deposited at the beginning of rifting of the Cameros basin during the late Jurassic-
Early Cretaceous; (2) to establish the development of source areas and provide a paleotectonic reconstruction of the sandstones of the Tera Group in the eastern sector of the basin; (3) to compare our results with sandstones signatures of the Tera Group in the western sector of the basin (Arribas et al., 2003); (4) to discuss provenance models for intraplate rift basins through a comparison with modern rifted-margin sands of the Red Sea and Gulf of Aden (Garzanti et al., 2001, 2003); and (5) to contribute to a global data base of petrographic provenance data for intra-plate rift basins.

\section{Geological and stratigraphic setting}

The Cameros Basin in the northern Iberian Range (Fig. 1) forms part of the Mesozoic Iberian Rift System (Mas et al., 1993; Guimerà et al., 1995; Salas et al., 2001; Mas et al., 2002, 2003). Intraplate rifting was a consequence of the opening of the oceanic Bay of Biscay, which separated Iberia from Europe. The Cameros Basin is the westernmost basin of the Mesozoic Iberian Rift System. It shows several atypical features including scarce marine influence and high subsidence and sediment accumulation rates. Another main feature of this basin is

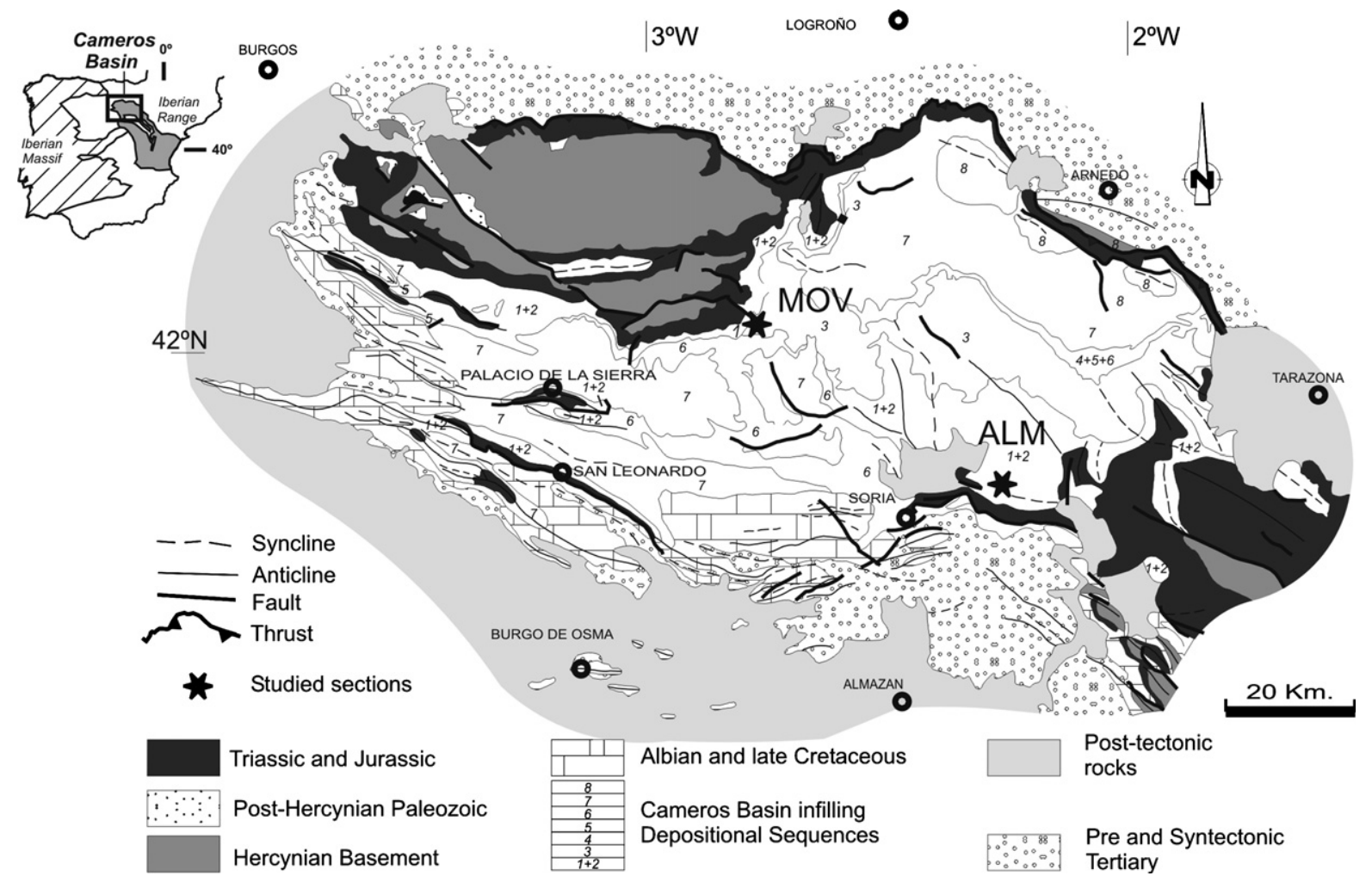

Fig. 1. Geological map of the Cameros Basin indicating the sections: MOV, Montenegro-Villoslada en Cameros (northwestern section) and ALM, Almajano (southeastern section). Modified from Guimerà et al. (1995). 


\section{CAMEROS BASIN}

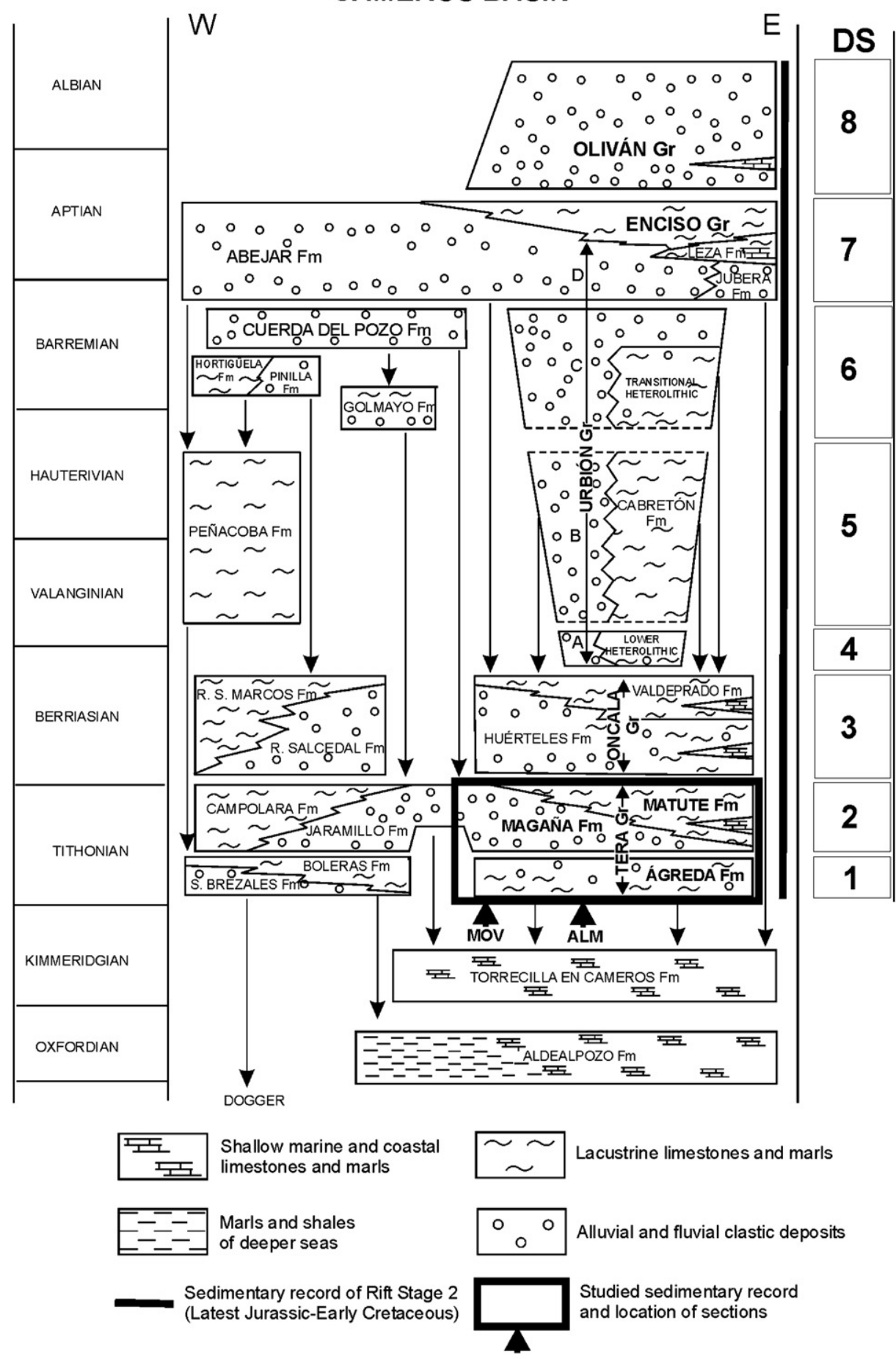

Fig. 2. Stratigraphy of the depositional sequences (DS) of the Cameros Basin. The stratigraphic interval examined is indicated (Tera Group, DS 1 and DS 2). MOV, Montenegro-Villoslada en Cameros (northwestern section) and ALM, Almajano (southeastern section). Modified from Mas et al. (2004).

that a large proportion of the Upper Jurassic-Lower Cretaceous deposits of the northwestern area are affected by low to very low-grade hydrothermal metamorphism (e.g. Guiraud and Seguret, 1985; Casquet et al., 1992; Alonso-Azcarate et al., 1995, 1999; Barrenechea et al., 2000; Mas et al., 2003). 
The basin-fill succession of the Cameros Basin embodies a large cycle or megasequence composed of up to $5000 \mathrm{~m}$ of sediment deposited from the Tithonian to early Albian. These deposits overlie late Jurassic marine carbonates, commonly those of the Kimmeridgian Torrecilla en Cameros Formation, and are separated from them by an erosional unconformity with associated paleosols and/or paleokarst features (Alonso and Mas, 1990; Benito, 2001; Benito and Mas, 2002; Benito et al., 2005). This socalled infra-Tithonian unconformity is present throughout the basin and occurs at the base of the Tera Group, which is the subject of this study. The stratigraphic gap represented by this unconformity is more important in the northern part of the basin than in the central and southern areas. In the northern area of the basin, it extends from the Late Kimmeridgian to part of the Barremian. Towards the center and south, it generally extends from the Late Kimmeridgian to the Early Tithonian.

The sedimentary infill of the Cameros Basin has been divided into eight depositional sequences (Mas et al., 2002, 2003) spanning the Tithonian to early Albian (Fig. 2). This sedimentary record consists of continental sediments corresponding to alluvial and lacustrine systems, with very rare marine incursions (Mas et al., 1993; Gómez Fernández and Meléndez, 1994). The Tera Group represents the first stage of rifting and is formed by two depositional sequences (DS 1 and DS 2, Fig. 2), which are Tithonian in age (Mas et al., 1993, Martín-Closas and Alonso Millán, 1998; Mas et al., 2004). The thickness and lateral continuity of DS 1 are highly variable. This depositional sequence is represented by clastic alluvial facies and lacustrine-palustrine carbonate facies. In contrast, DS 2 is especially thick, up to $1500 \mathrm{~m}$ in the depocenter, and consists of a clastic fluvial facies which grades upwards and laterally to carbonate lacustrine facies.

Arribas et al. $(2002,2003)$ characterized the basin-fill successions of the western sector of the Cameros Basin in terms of their clastic constituents and sandstone sources. These authors identified four main petrofacies (A, B, C and D) from DS 1 to DS 7. Petrofacies A is quartzo-sedimentolithic (mean: $\mathrm{Qm}_{85} \mathrm{~F}_{2} \mathrm{Lt}_{13}$ ) and characterizes DS 1. This petrofacies records the erosion of Jurassic marine pre-rift cover. Petrofacies B is quartzofeldspathic (mean: $\mathrm{Qm}_{81} \mathrm{~F}_{14} \mathrm{Lt}_{5}$ ) and is derived from the erosion of metamorphic terranes of the West-Asturian Leonese Zone during the deposition of DS-2 and DS-3. The quartzarenitic Petrofacies C (mean: $\mathrm{Qm}_{95} \mathrm{~F}_{3} \mathrm{Lt}_{2}$ ) was generated by recycling of the sedimentary cover, mainly Triassic arkoses and carbonate rocks. Finally, Petrofacies D is quartzofeldspathic (mean: $\mathrm{Qm}_{88} \mathrm{~F}_{11} \mathrm{Lt}_{1}$ ), and attributed to erosion of coarse crystalline plutonic rocks from the Central Iberian Zone.
Analysis of sandstone provenance in the eastern sector of the Cameros Basin has received less attention. The present paper focuses on the beginning of rifting (Tera Group) in this eastern sector.

Two representative stratigraphic sections of the Tera Group in the eastern sector of the basin were examined (Fig. 1): one located in the northwest (MontenegroVilloslada, MOV, $377 \mathrm{~m}$ thick) and the other in the southeast of the study area (Almajano, ALM, $343 \mathrm{~m}$ thick). Based on illite and chlorite crystallinity data (Barrenechea et al., 2001), the Tera Group deposits of the Montenegro-Villoslada section attained low-anchizonal conditions, whereas the Almajano section deposits only suffered diagenetic conditions.

In both study areas, the Tera Group can be subdivided into three formations: Ágreda, Magaña and Sierra de Matute (Mas et al., 1993; Gómez Fernández and Meléndez, 1994) (Figs. 2 and 3). The main sedimentological characteristics of DS 1 and DS 2 in the sections examined can be summarized as follows:

Depositional sequence 1

Ágreda Fm. (DS 1). In the southeastern section (ALM), the Ágreda Fm. is composed of conglomerates including abundant carbonate-rock fragments that pass upwards into coarse-medium grained sandstones with trough cross bedding, interbedded with reddish mudstones containing calcrete profiles. The Ágreda Fm. corresponds to a distal system of alluvial fans with substantial development of meandering channels and mud flats. These alluvial fans are related to the onset of rifting. A high rate of vertical accretion is indicated by the high ratio of floodplain to channel facies. In the northwestern section (MOV), this formation is composed of nodular limestones, with root marks and a bed of calcareous conglomerate. The Ágreda Fm. was deposited in a palustrine environment and has abundant pedogenic features. Its thickness varies from $7 \mathrm{~m}$ in the northwester section to $78 \mathrm{~m}$ in the southeastern section (Fig. 3).

Depositional sequence 2

Magaña Fm (DS 2). This formation is composed of channel-fill (sandy point-bars) and crevasse deposits, interbedded with floodplain mudstones displaying abundant paleosols. Sandstones appear generally as sheets less than $5 \mathrm{~m}$ thick. The Magaña Fm. was deposited in a meandering fluvial system. In the southeastern section (ALM), it attains a thickness of $121 \mathrm{~m}$ and contains some conglomeratic channel fills (Fig. 3). In the northwestern section (MOV), the formation is thicker $(370 \mathrm{~m})$, and can be divided into Magaña Fms. 1 and 2 (Fig. 3). Channel facies are more common in Magaña Fm. 1 and, occasionally, amalgamated channel-fill and crevasse deposits 

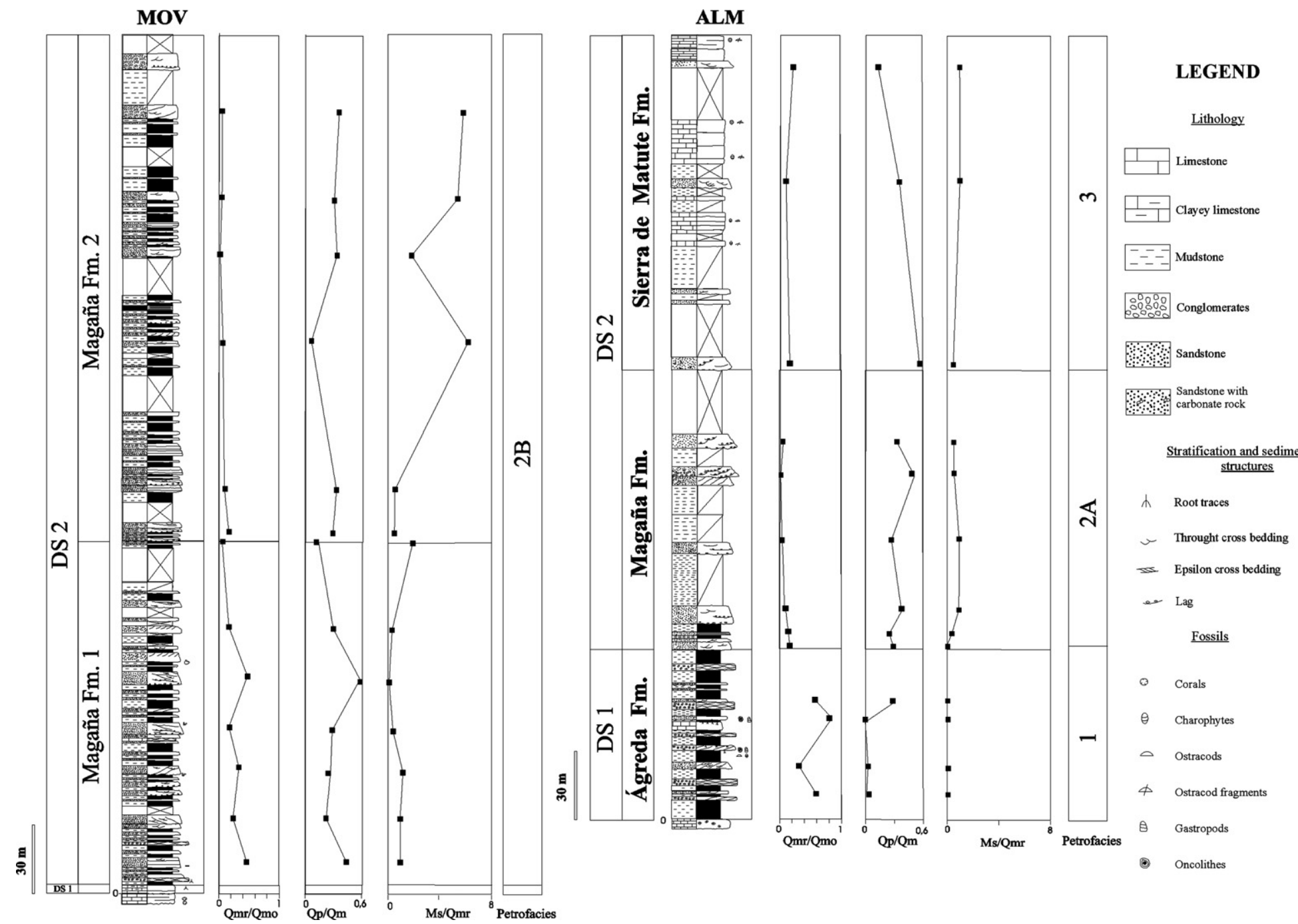

Fig. 3. Stratigraphic sections of Almajano (southeastern section) and Montenegro-Villoslada (northwestern section). The Qmr/Qmo, the Qp/Qm and the Ms/Qmr ratios are represented in each section. Qmr: monocrystalline quartz, undulosity $<5^{\circ}$; Qmo: Monocrystalline quartz, undulosity $>5^{\circ}$; Qp: polycrystalline quartz; Qm: Monocrystalline quartz; Ms: muscovite. 
Table 1

Petrographic data base of sandstones from Almajano section (ALM)

\begin{tabular}{|c|c|c|c|c|c|c|c|c|c|c|c|c|c|c|c|c|c|c|c|c|c|c|c|c|c|c|c|c|c|}
\hline & & & & \multicolumn{2}{|c|}{ ALM-101 } & \multicolumn{2}{|c|}{ ALM-102 } & \multicolumn{2}{|c|}{ ALM-103 } & \multicolumn{2}{|c|}{ ALM-104 } & \multicolumn{2}{|c|}{ ALM-105 } & \multicolumn{2}{|c|}{ ALM-106 } & \multicolumn{2}{|c|}{ ALM-107 } & \multicolumn{2}{|c|}{ ALM-108 } & \multicolumn{2}{|c|}{ ALM-109 } & \multicolumn{2}{|c|}{ ALM-110 } & \multicolumn{2}{|c|}{ ALM-111 } & \multicolumn{2}{|c|}{ ALM-112 } & \multicolumn{2}{|c|}{ ALM-113 } \\
\hline & & & & $\mathrm{N}$ & $\%$ & $\mathrm{~N}$ & $\%$ & $\mathrm{~N}$ & $\%$ & $\mathrm{~N}$ & $\%$ & $\mathrm{~N}$ & $\%$ & $\mathrm{~N}$ & $\%$ & $\mathrm{~N}$ & $\%$ & $\mathrm{~N}$ & $\%$ & $\mathrm{~N}$ & $\%$ & $\mathrm{~N}$ & $\%$ & $\mathrm{~N}$ & $\%$ & $\mathrm{~N}$ & $\%$ & $\mathrm{~N}$ & $\%$ \\
\hline \multirow[t]{19}{*}{ NCE } & $\mathrm{Q}$ & Qmr & $\begin{array}{l}\text { Monocrystalline quartz, } \\
\text { undulosity }<5^{\circ}\end{array}$ & 30 & 6.6 & 31 & 7.6 & 4 & 2.6 & 46 & 9.3 & 23 & 5.0 & 19 & 4.7 & 13 & 3.2 & 10 & 2.5 & 3 & 0.7 & 9 & 2.2 & 27 & 5.8 & 8 & 1.6 & 29 & 6.3 \\
\hline & & Qmo & $\begin{array}{l}\text { Monocrystalline quartz, } \\
\text { undulosity }>5^{\circ}\end{array}$ & 50 & 11.0 & 101 & 24.8 & 5 & 3.3 & 81 & 16.4 & 159 & 34.4 & 144 & 35.8 & 228 & 56.2 & 195 & 48.5 & 214 & 53.0 & 187 & 46.6 & 186 & 40.1 & 161 & 32.1 & 131 & 28.4 \\
\hline & & $\mathrm{Qm}[\mathrm{Q}]$ & $\begin{array}{l}\text { Monocrystalline } \\
\text { quartz with inherited } \\
\text { sintaxial cement }\end{array}$ & 20 & 4.4 & 10 & 2.5 & & 0.0 & 1 & 0.2 & & 0.0 & & 0.0 & & 0.0 & & 0.0 & & 0.0 & & 0.0 & & 0.0 & & 0.0 & & 0.0 \\
\hline & & Qp2-3 & $\begin{array}{l}\text { Polycrystalline quartz } \\
\text { with 2-3 subgrains }\end{array}$ & 5 & 1.1 & 2 & 0.5 & & 0.0 & 25 & 5.1 & 16 & 3.5 & 23 & 5.7 & 19 & 4.7 & 16 & 4.0 & 8 & 2.0 & 25 & 6.2 & 17 & 3.7 & 22 & 4.4 & 20 & 4.3 \\
\hline & & $\mathrm{Qp}>3$ & $\begin{array}{l}\text { Polycrystalline quartz } \\
>3 \text { subgrains }\end{array}$ & & 0.0 & & 0.0 & & 0.0 & 8 & 1.6 & 42 & 9.1 & 36 & 9.0 & 35 & 8.6 & 62 & 15.4 & 54 & 13.4 & 73 & 18.2 & 51 & 11.0 & 77 & 15.3 & 44 & 9.5 \\
\hline & & Qfrg & $\begin{array}{l}\text { Quartz in plutonic } \\
\text { rock fragment }\end{array}$ & & 0.0 & & 0.0 & & 0.0 & & 0.0 & & 0.0 & 14 & 3.5 & 7 & 1.7 & 11 & 2.7 & 1 & 0.2 & 19 & 4.7 & & 0.0 & & 0.0 & & 0.0 \\
\hline & & $\mathrm{Cq}$ & $\begin{array}{l}\text { Carbonate replacement } \\
\text { on quartz }\end{array}$ & 10 & 2.2 & 6 & 1.5 & & 0.0 & 28 & 5.7 & 18 & 3.9 & 3 & 0.7 & 3 & 0.7 & & 0.0 & & 0.0 & 1 & 0.2 & 11 & 2.4 & 13 & 2.6 & 21 & 4.5 \\
\hline & $\mathrm{K}$ & Ks & $\begin{array}{l}\text { K-feldspar, } \\
\text { single crystals }\end{array}$ & 1 & 0.2 & 5 & 1.2 & & 0.0 & 6 & 1.2 & 9 & 1.9 & 13 & 3.2 & 26 & 6.4 & 19 & 4.7 & 34 & 8.4 & 12 & 3.0 & 8 & 1.7 & 8 & 1.6 & 9 & 1.9 \\
\hline & & Kfrg & $\begin{array}{l}\text { K-feldspar in coarse- } \\
\text { grained rock fragment }\end{array}$ & & 0.0 & & 0.0 & & 0.0 & & 0.0 & & 0.0 & & 0.0 & & 0.0 & 5 & 1.2 & 2 & 0.5 & 7 & 1.7 & & 0.0 & & 0.0 & & 0.0 \\
\hline & & $\mathrm{CaoK}$ & $\begin{array}{l}\text { Kaolinite replacement } \\
\text { K-feldspar }\end{array}$ & & 0.0 & & 0.0 & & 0.0 & & 0.0 & & 0.0 & 6 & 1.5 & 9 & 2.2 & 25 & 6.2 & 7 & 1.7 & 21 & 5.2 & & 0.0 & & 0.0 & & 0.0 \\
\hline & & Cik & $\begin{array}{l}\text { Kaolinite plus illite } \\
\text { replacement K-feldspar }\end{array}$ & & 0.0 & & 0.0 & & 0.0 & & 0.0 & & 0.0 & 2 & 0.5 & 1 & 0.2 & 10 & 2.5 & 2 & 0.5 & 3 & 0.7 & & 0.0 & 1 & 0.2 & & 0.0 \\
\hline & & Kil & $\begin{array}{l}\text { Illite replacement } \\
\text { K-feldspar }\end{array}$ & & 0.0 & & 0.0 & & 0.0 & & 0.0 & 2 & 0.4 & 3 & 0.7 & 3 & 0.7 & & 0.0 & & 0.0 & 3 & 0.7 & 5 & 1.1 & 2 & 0.4 & 5 & 1.1 \\
\hline & & $\mathrm{Ck}$ & $\begin{array}{l}\text { Carbonate replacement } \\
\text { on K-feldspar }\end{array}$ & 2 & 0.4 & 1 & 0.2 & & 0.0 & & 0.0 & 1 & 0.2 & 2 & 0.5 & 5 & 1.2 & & 0.0 & 19 & 4.7 & 1 & 0.2 & 9 & 1.9 & 1 & 0.2 & 4 & 0.9 \\
\hline & $\mathrm{P}$ & Ps & $\begin{array}{l}\text { Plagioclase, } \\
\text { single crystals }\end{array}$ & & 0.0 & & 0.0 & & 0.0 & & 0.0 & & 0.0 & & 0.0 & & 0.0 & 2 & 0.5 & & 0.0 & & 0.0 & 2 & 0.4 & 1 & 0.2 & 1 & 0.2 \\
\hline & & $\mathrm{Ab}$ & Albite & 1 & 0.2 & 7 & 1.7 & & 0.0 & 3 & 0.6 & 3 & 0.6 & 20 & 5.0 & 2 & 0.5 & & 0.0 & & 0.0 & & 0.0 & 21 & 4.5 & 55 & 11.0 & 43 & 9.3 \\
\hline & & Ail & $\begin{array}{l}\text { Illite replacement } \\
\text { on albite }\end{array}$ & & 0.0 & & 0.0 & & 0.0 & & 0.0 & & 0.0 & & 0.0 & & 0.0 & & 0.0 & & 0.0 & & 0.0 & 4 & 0.9 & 3 & 0.6 & & 0.0 \\
\hline & & $\mathrm{Cab}$ & $\begin{array}{l}\text { Carbonate replacement } \\
\text { on Plagioclase }\end{array}$ & & 0.0 & & 0.0 & & 0.0 & & 0.0 & & 0.0 & & 0.0 & & 0.0 & & 0.0 & & 0.0 & & 0.0 & 5 & 1.1 & 5 & 1.0 & 5 & 1.1 \\
\hline & $\mathrm{L}$ & $\mathrm{Ch}$ & Chert & & 0.0 & & 0.0 & & 0.0 & & 0.0 & 8 & 1.7 & 1 & 0.2 & 2 & 0.5 & 2 & 0.5 & 5 & 1.2 & 2 & 0.5 & 8 & 1.7 & 6 & 1.2 & 4 & 0.9 \\
\hline & & $\mathrm{Lm}$ & Schist-Slate & & 0.0 & & 0.0 & & 0.0 & & 0.0 & 1 & 0.2 & 3 & 0.7 & & 0.0 & & 0.0 & & 0.0 & & 0.0 & 1 & 0.2 & 1 & 0.2 & 2 & 0.4 \\
\hline
\end{tabular}




\begin{tabular}{|c|c|c|c|c|c|c|c|c|c|c|c|c|c|c|c|c|c|c|c|c|c|c|c|c|c|c|c|c|}
\hline & & & \multicolumn{2}{|c|}{ ALM-101 } & \multicolumn{2}{|c|}{ ALM-102 } & \multicolumn{2}{|c|}{ ALM-103 } & \multicolumn{2}{|c|}{ ALM-104 } & \multicolumn{2}{|c|}{ ALM-105 } & \multicolumn{2}{|c|}{ ALM-106 } & \multicolumn{2}{|c|}{ ALM-107 } & \multicolumn{2}{|c|}{ ALM-108 } & \multicolumn{2}{|c|}{ ALM-109 } & \multicolumn{2}{|c|}{ ALM-110 } & \multicolumn{2}{|c|}{ ALM-111 } & \multicolumn{2}{|c|}{ ALM-112 } & \multicolumn{2}{|c|}{ ALM-113 } \\
\hline & & & $\mathrm{N}$ & $\%$ & $\mathrm{~N}$ & $\%$ & $\mathrm{~N}$ & $\%$ & $\mathrm{~N}$ & $\%$ & $\mathrm{~N}$ & $\%$ & $\mathrm{~N}$ & $\%$ & $\mathrm{~N}$ & $\%$ & $\mathrm{~N}$ & $\%$ & $\mathrm{~N}$ & $\%$ & $\mathrm{~N}$ & $\%$ & $\mathrm{~N}$ & $\%$ & $\mathrm{~N}$ & $\%$ & $\mathrm{~N}$ & $\%$ \\
\hline \multirow[t]{5}{*}{ M } & Ms & Muscovite & & 0.0 & & 0.0 & & 0.0 & & 0.0 & 2 & 0.4 & 10 & 2.5 & 3 & 0.7 & 9 & 2.2 & 2 & 0.5 & 5 & 1.2 & 12 & 2.6 & 8 & 1.6 & 25 & 5.4 \\
\hline & Mfrg & $\begin{array}{l}\text { Muscovite in coarse- } \\
\text { grained rock fragment }\end{array}$ & & 0.0 & & 0.0 & & 0.0 & & 0.0 & & 0.0 & 2 & 0.5 & & 0.0 & & 0.0 & & 0.0 & & 0.0 & & 0.0 & & 0.0 & & 0.0 \\
\hline & $\mathrm{Tu}$ & Tourmaline & 1 & 0.2 & & 0.0 & & 0.0 & & 0.0 & 1 & 0.2 & & 0.0 & 1 & 0.2 & 1 & 0.2 & & 0.0 & 1 & 0.2 & & 0.0 & 4 & 0.8 & 3 & 0.6 \\
\hline & Op & Opaque & & 0.0 & 14 & 3.4 & & 0.0 & 3 & 0.6 & & 0.0 & & 0.0 & & 0.0 & & 0.0 & & 0.0 & & 0.0 & & 0.0 & & 0.0 & & 0.0 \\
\hline & & & & 0.0 & & 0.0 & & 0.0 & & 0.0 & & 0.0 & & 0.0 & & 0.0 & & 0.0 & & 0.0 & & 0.0 & & 0.0 & & 0.0 & & 0.0 \\
\hline \multicolumn{2}{|c|}{ CE Ml } & Micritic limestone & 77 & 17.0 & 128 & 31.4 & & 0.0 & & 0.0 & 14 & 3.0 & & 0.0 & & 0.0 & & 0.0 & & 0.0 & & 0.0 & & 0.0 & & 0.0 & & 0.0 \\
\hline & $\mathrm{Sc}$ & Sparitic limestone & 22 & 4.9 & 17 & 4.2 & 4 & 2.6 & 6 & 1.2 & 22 & 4.8 & & 0.0 & & 0.0 & & 0.0 & & 0.0 & & 0.0 & 35 & 7.5 & 2 & 0.4 & 5 & 1.1 \\
\hline & $\mathrm{Md}$ & Dolomicrite & 21 & 4.6 & 9 & 2.2 & 42 & 27.5 & 49 & 9.9 & 13 & 2.8 & & 0.0 & & 0.0 & & 0.0 & & 0.0 & & 0.0 & 1 & 0.2 & 6 & 1.2 & 46 & 10.0 \\
\hline & $\mathrm{Sd}$ & Dolosparite & 14 & 3.1 & 1 & 0.2 & & 0.0 & 2 & 0.4 & 1 & 0.2 & & 0.0 & & 0.0 & & 0.0 & & 0.0 & & 0.0 & & 0.0 & 2 & 0.4 & 11 & 2.4 \\
\hline & Afrc & $\begin{array}{l}\text { Ankerite replacement } \\
\text { on carbonate rock } \\
\text { fragment }\end{array}$ & 10 & 2.2 & & 0.0 & & 0.0 & & 0.0 & 8 & 1.7 & & 0.0 & & 0.0 & & 0.0 & & 0.0 & & 0.0 & 1 & 0.2 & 55 & 11.0 & 18 & 3.9 \\
\hline & Fo & Fossils & 1 & 0.2 & 1 & 0.2 & & 0.0 & & 0.0 & & 0.0 & & 0.0 & & 0.0 & & 0.0 & & 0.0 & & 0.0 & & 0.0 & & 0.0 & 1 & 0.2 \\
\hline \multirow{2}{*}{\multicolumn{2}{|c|}{ Ep }} & Echinoderm plates & 8 & 1.8 & 13 & 3.2 & & 0.0 & 2 & 0.4 & & 0.0 & & 0.0 & & 0.0 & & 0.0 & & 0.0 & & 0.0 & 3 & 0.6 & & 0.0 & & 0.0 \\
\hline & & & & 0.0 & & 0.0 & & 0.0 & & 0.0 & & 0.0 & & 0.0 & & 0.0 & & 0.0 & & 0.0 & & 0.0 & & 0.0 & 7 & 1.4 & 1 & 0.2 \\
\hline \multicolumn{2}{|c|}{ NCIIn } & Intraclast & 1 & 0.2 & 2 & 0.5 & 2 & 1.3 & 3 & 0.6 & & 0.0 & & 0.0 & & 0.0 & & 0.0 & & 0.0 & & 0.0 & & 0.0 & & 0.0 & & 0.0 \\
\hline \multirow[t]{4}{*}{$\mathrm{CI}$} & Mli & Micritic limestone & & 0.0 & & 0.0 & 83 & 54.2 & 140 & 28.3 & & 0.0 & & 0.0 & & 0.0 & & 0.0 & & 0.0 & & 0.0 & & 0.0 & & 0.0 & & 0.0 \\
\hline & & $\begin{array}{l}\text { TOTAL } \\
\text { FRAMEWORK }\end{array}$ & 274 & 60.5 & 348 & 85.5 & 140 & 91.5 & 403 & 81.4 & 343 & 74.2 & 301 & 74.9 & 357 & 87.9 & 367 & 91.3 & 351 & 86.9 & 369 & 92.0 & 407 & 87.7 & 448 & 89.2 & 428 & 92.6 \\
\hline & {$[\mathrm{Ca}]$} & Calcite cement & & 0.0 & 10 & 2.5 & 4 & 2.6 & 39 & 7.9 & 7 & 1.5 & & 0.0 & & 0.0 & & 0.0 & & 0.0 & & 0.0 & 8 & 1.7 & 1 & 0.2 & & 0.0 \\
\hline & {$[\mathrm{Dol}]$} & Dolomite cement & & 0.0 & 4 & 1.0 & & 0.0 & 12 & 2.4 & 6 & 1.3 & & 0.0 & & 0.0 & & 0.0 & & 0.0 & & 0.0 & 18 & 3.9 & 34 & 6.8 & 3 & 0.6 \\
\hline $\mathrm{C}$ & [Anq] & Anque & & 0.0 & 19 & 4.7 & & 0.0 & 9 & 1.8 & 15 & 3.2 & 10 & 2.5 & 6 & 1.5 & 4 & 1.0 & 6 & 1.5 & & 0.0 & 4 & 0.9 & & 0.0 & 10 & 2.2 \\
\hline $\mathrm{E}$ & {$[\mathrm{Q}]$} & Quartz cement & & 0.0 & 18 & 4.4 & & 0.0 & 26 & 5.3 & 38 & 8.2 & 26 & 6.5 & 20 & 4.9 & 10 & 2.5 & 33 & 8.2 & 23 & 5.7 & 9 & 1.9 & 14 & 2.8 & 17 & 3.7 \\
\hline M & Ank[Q] & $\begin{array}{l}\text { Anquerite replacing } \\
\text { quartz cement }\end{array}$ & & & & & & 0.0 & & 0.0 & 4 & 0.9 & 4 & 1.0 & & 0.0 & & 0.0 & & 0.0 & & 0.0 & 2 & 0.4 & & 0.0 & 2 & 0.4 \\
\hline $\mathrm{E}$ & {$[\mathrm{Cao}]$} & Kaolinite cement & & 0.0 & & 0.0 & & 0.0 & & 0.0 & 27 & 5.8 & 17 & 4.2 & 16 & 3.9 & 5 & 1.2 & 3 & 0.7 & 8 & 2.0 & 10 & 2.2 & & 0.0 & & 0.0 \\
\hline $\mathrm{N}$ & $\mathrm{Anq}[\mathrm{Cao}]$ & $\begin{array}{l}\text { Anquerite replacing } \\
\text { kaolinite cement }\end{array}$ & & 0.0 & & 0.0 & & 0.0 & & 0.0 & 4 & 0.9 & 3 & 0.7 & & 0.0 & & 0.0 & & 0.0 & & 0.0 & & 0.0 & & 0.0 & & 0.0 \\
\hline $\mathrm{T}$ & [Cao-il] & Kaolinite-illite cement & & 0.0 & & 0.0 & & 0.0 & & 0.0 & & 0.0 & & 0.0 & & 0.0 & & 0.0 & & 0.0 & & 0.0 & & 0.0 & & 0.0 & & 0.0 \\
\hline \multirow[t]{4}{*}{$\mathrm{S}$} & [il] & Illite cement & & 0.0 & & 0.0 & & 0.0 & & 0.0 & & 0.0 & 1 & 0.2 & & 0.0 & & 0.0 & & 0.0 & & 0.0 & & 0.0 & & 0.0 & & 0.0 \\
\hline & {$[\mathrm{Fe}]$} & $\mathrm{Fe}$-oxide cement & & 0.0 & 7 & 1.7 & 7 & 4.6 & 5 & 1.0 & 18 & 3.9 & 40 & 10.0 & 7 & 1.7 & 16 & 4.0 & 9 & 2.2 & 1 & 0.2 & 6 & 1.3 & 5 & 1.0 & 2 & 0.4 \\
\hline & {$[\mathrm{K}]$} & K-feldspar cement & & 0.0 & 1 & 0.2 & & 0.0 & 1 & 0.2 & & 0.0 & & 0.0 & & 0.0 & & 0.0 & 2 & 0.5 & & 0.0 & & 0.0 & & 0.0 & & 0.0 \\
\hline & & TOTAL & 274 & 60.5 & 407 & 100.0 & 153 & 100.0 & 495 & 100.0 & 462 & 100.0 & 402 & 100.0 & 406 & 100.0 & 402 & 100.0 & 404 & 100.0 & 401 & 100.0 & 464 & 100.0 & 502 & 100.0 & 462 & 100.0 \\
\hline
\end{tabular}

Quartz typologies have been classified following the criteria of Basu et al. (1975). Plutonic rock fragments have been counted as quartz, feldspar and muscovite in plutonic rock fragments (Qfrg, Kfrg, Mfrg). 
Table 2

Petrographic data base of sandstones from Montenegro-Villoslada section (MOV)

\begin{tabular}{|c|c|c|c|c|c|c|c|c|c|c|c|c|c|c|c|c|c|c|c|c|c|c|c|c|c|c|c|c|c|}
\hline & & & & \multicolumn{2}{|c|}{ MOV-100 } & \multicolumn{2}{|c|}{ MOV-101 } & \multicolumn{2}{|c|}{ MOV-102 } & \multicolumn{2}{|c|}{ MOV-103 } & \multicolumn{2}{|c|}{ MOV-104 } & \multicolumn{2}{|c|}{ MOV-105 } & \multicolumn{2}{|c|}{ MOV-106 } & \multicolumn{2}{|c|}{ MOV-107 } & \multicolumn{2}{|c|}{ MOV-108 } & \multicolumn{2}{|c|}{ MOV-109 } & \multicolumn{2}{|c|}{ MOV-110 } & \multicolumn{2}{|c|}{ MOV-111 } & \multicolumn{2}{|c|}{ MOV-112 } \\
\hline & & & & $\mathrm{N}$ & $\%$ & $\mathrm{~N}$ & $\%$ & $\mathrm{~N}$ & $\%$ & $\mathrm{~N}$ & $\%$ & $\mathrm{~N}$ & $\%$ & $\mathrm{~N}$ & $\%$ & $\mathrm{~N}$ & $\%$ & $\mathrm{~N}$ & $\%$ & $\mathrm{~N}$ & $\%$ & $\mathrm{~N}$ & $\%$ & $\mathrm{~N}$ & $\%$ & $\mathrm{~N}$ & $\%$ & $\mathrm{~N}$ & $\%$ \\
\hline \multirow[t]{17}{*}{ NCE } & Q & Qmr & $\begin{array}{l}\text { Monocrystalline } \\
\text { quartz, undulosity }<5^{\circ}\end{array}$ & 45 & 11.2 & 24 & 5.9 & 27 & 4.4 & 23 & 5.9 & 51 & 12.7 & 24 & 6.0 & 8 & 2.0 & 26 & 6.1 & 14 & 3.4 & 11 & 2.9 & 3 & 0.7 & 10 & 2.3 & 9 & 2.2 \\
\hline & & Qmo & $\begin{array}{l}\text { Monocrystalline } \\
\text { quartz, undulosity }>5^{\circ}\end{array}$ & 101 & 25.1 & 104 & 25.7 & 81 & 13.1 & 142 & 36.5 & 108 & 26.8 & 145 & 36.3 & 173 & 42.9 & 150 & 35.3 & 158 & 38.6 & 175 & 46.2 & 166 & 41.2 & 185 & 42.5 & 151 & 36.7 \\
\hline & & $\begin{array}{l}\text { Qm } \\
{[\mathrm{Q}]}\end{array}$ & $\begin{array}{l}\text { Monocrystalline quartz } \\
\text { with inherited } \\
\text { sintaxial cement }\end{array}$ & & 0.0 & 8 & 2.0 & 4 & 0.6 & 2 & 0.5 & & 0.0 & & 0.0 & & 0.0 & & 0.0 & & 0.0 & & 0.0 & & 0.0 & & 0.0 & & 0.0 \\
\hline & & Qp2-3 & $\begin{array}{l}\text { Polycrystalline quartz } \\
\text { with 2-3 subgrains }\end{array}$ & 15 & 3.7 & 13 & 3.2 & 11 & 1.8 & 7 & 1.8 & 35 & 8.7 & 23 & 5.8 & 6 & 1.5 & 20 & 4.7 & 33 & 8.1 & 13 & 3.4 & 7 & 1.7 & 30 & 6.9 & 22 & 5.3 \\
\hline & & $\mathrm{Qp}>3$ & $\begin{array}{l}\text { Polycrystalline quartz } \\
>3 \text { subgrains }\end{array}$ & 46 & 11.4 & 12 & 3.0 & 14 & 2.3 & 39 & 10.0 & 54 & 13.4 & 25 & 6.3 & 18 & 4.5 & 27 & 6.4 & 26 & 6.4 & & 0.0 & 46 & 11.4 & 30 & 6.9 & 31 & 7.5 \\
\hline & & Qfrg & $\begin{array}{l}\text { Quartz in plutonic } \\
\text { rock fragment }\end{array}$ & & 0.0 & & 0.0 & & 0.0 & & 0.0 & & 0.0 & & 0.0 & & 0.0 & & 0.0 & 9 & 2.2 & & 0.0 & 12 & 3.0 & 24 & 5.5 & 1 & 0.2 \\
\hline & & $\mathrm{Cq}$ & $\begin{array}{l}\text { Carbonate replacement } \\
\text { on quartz }\end{array}$ & 3 & 0.7 & 7 & 1.7 & 3 & 0.5 & 2 & 0.5 & & 0.0 & 10 & 2.5 & 3 & 0.7 & 8 & 1.9 & 1 & 0.2 & 12 & 3.2 & & 0.0 & 2 & 0.5 & 3 & 0.7 \\
\hline & $\mathrm{K}$ & Ks & $\begin{array}{l}\text { K-feldspar, } \\
\text { single crystals }\end{array}$ & 32 & 8.0 & 6 & 1.5 & & 0.0 & 24 & 6.2 & 44 & 10.9 & 48 & 12.0 & 50 & 12.4 & 64 & 15.1 & 65 & 15.9 & 16 & 4.2 & 61 & 15.1 & 43 & 9.9 & 67 & 16.3 \\
\hline & & Kfrg & $\begin{array}{l}\text { K-feldspar in coarse- } \\
\text { grained rock fragment }\end{array}$ & & 0.0 & & 0.0 & & 0.0 & & 0.0 & & 0.0 & & 0.0 & & 0.0 & & 0.0 & 6 & 1.5 & & 0.0 & 2 & 0.5 & & 0.0 & 1 & 0.2 \\
\hline & & CaoK & $\begin{array}{l}\text { Kaolinite replacement } \\
\text { K-feldspar }\end{array}$ & & 0.0 & & 0.0 & & 0.0 & & 0.0 & & 0.0 & & 0.0 & & 0.0 & & 0.0 & & 0.0 & & 0.0 & & 0.0 & & 0.0 & & 0.0 \\
\hline & & Cik & $\begin{array}{l}\text { Kaolinite plus illite } \\
\text { replacement K-feldspar }\end{array}$ & 3 & 0.7 & & 0.0 & & 0.0 & & 0.0 & & 0.0 & & 0.0 & 2 & 0.5 & & 0.0 & 5 & 1.2 & & 0.0 & 17 & 4.2 & 1 & 0.2 & & 0.0 \\
\hline & & Kil & $\begin{array}{l}\text { Illite replacement } \\
\text { K-feldspar }\end{array}$ & 3 & 0.7 & 4 & 1.0 & & 0.0 & 2 & 0.5 & & 0.0 & 1 & 0.3 & & 0.0 & 8 & 1.9 & 7 & 1.7 & & 0.0 & & 0.0 & 3 & 0.7 & 13 & 3.2 \\
\hline & & $\mathrm{Ck}$ & $\begin{array}{l}\text { Carbonate replacement } \\
\text { on K-feldspar }\end{array}$ & 1 & 0.2 & 2 & 0.5 & 9 & 1.5 & 3 & 0.8 & 2 & 0.5 & 12 & 3.0 & & 0.0 & 7 & 1.6 & 2 & 0.5 & 1 & 0.3 & 1 & 0.2 & & 0.0 & 7 & 1.7 \\
\hline & $\mathrm{P}$ & Ps & $\begin{array}{l}\text { Plagioclase, } \\
\text { single crystals }\end{array}$ & 5 & 1.2 & 3 & 0.7 & & 0.0 & 5 & 1.3 & 9 & 2.2 & 1 & 0.3 & 5 & 1.2 & 5 & 1.2 & 6 & 1.5 & & 0.0 & 3 & 0.7 & 5 & 1.1 & 3 & 0.7 \\
\hline & & $\mathrm{Ab}$ & Albite & 23 & 5.7 & 35 & 8.7 & 30 & 4.8 & 22 & 5.7 & 18 & 4.5 & 6 & 1.5 & 12 & 3.0 & 12 & 2.8 & 10 & 2.4 & 21 & 5.5 & 9 & 2.2 & 6 & 1.4 & 18 & 4.4 \\
\hline & & Ail & $\begin{array}{l}\text { Illite replacement } \\
\text { on albite }\end{array}$ & & 0.0 & & 0.0 & 6 & 1.0 & & 0.0 & 2 & 0.5 & 1 & 0.3 & & 0.0 & & 0.0 & & 0.0 & & 0.0 & & 0.0 & & 0.0 & & 0.0 \\
\hline & & $\mathrm{Cab}$ & $\begin{array}{l}\text { Carbonate replacement } \\
\text { on Plagioclase }\end{array}$ & 1 & 0.2 & 4 & 1.0 & 2 & 0.3 & 1 & 0.3 & 1 & 0.2 & 3 & 0.8 & & 0.0 & & 0.0 & & 0.0 & & 0.0 & & 0.0 & & 0.0 & & 0.0 \\
\hline
\end{tabular}




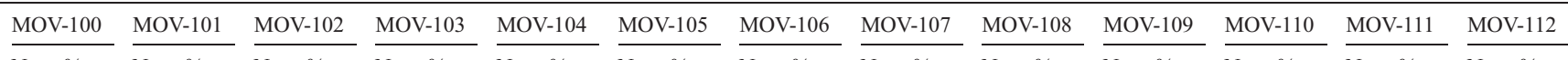

$\begin{array}{lllrrrrrrrrrrrrrrrrrrrrrrrrrrrrrrrr} & \text { L } & \text { Ch } & \text { Chert } & 11 & 2.7 & 8 & 2.0 & 3 & 0.5 & 5 & 1.3 & 7 & 1.7 & 8 & 2.0 & 11 & 2.7 & 8 & 1.9 & 10 & 2.4 & 2 & 0.5 & 5 & 1.2 & 5 & 1.1 & 6 & 1.5 \\ & \text { Lm } & \text { Schist-Slate } & 7 & 1.7 & 2 & 0.5 & 2 & 0.3 & 3 & 0.8 & 2 & 0.5 & 1 & 0.3 & 8 & 2.0 & 4 & 0.9 & 5 & 1.2 & & 0.0 & 2 & 0.5 & 5 & 1.1 & & 0.0\end{array}$

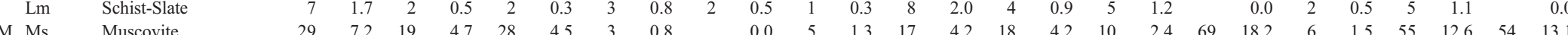

$\begin{array}{lllllllllllllllllllllllllll}\text { M Ms } & \text { Muscovite } & 29 & 7.2 & 19 & 4.7 & 28 & 4.5 & 3 & 0.8 & 0.0 & 5 & 1.3 & 17 & 4.2 & 18 & 4.2 & 10 & 2.4 & 69 & 18.2 & 6 & 1.5 & 55 & 12.6 & 54 & 13.1\end{array}$

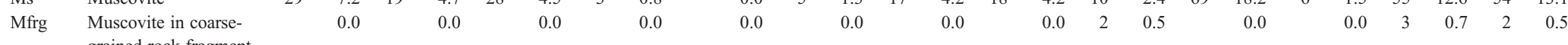

$\begin{array}{lllllllllllllllllllll}\mathrm{Tu} & \text { Tourmaline } & 1 & 0.2 & 3 & 0.7 & 1 & 0.2 & 0.0 & 0.0 & 1 & 0.3 & 0.0 & 0.0 & 0.0 & 1 & 0.3 & 0.0 & 1 & 0.2 & 0.0\end{array}$

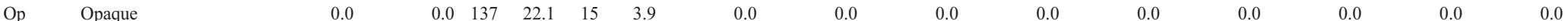

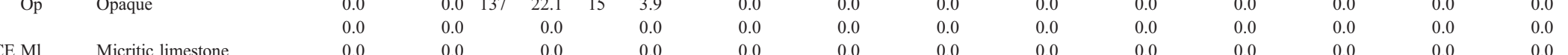

$\begin{array}{lllllllllllllll}\text { CE Ml Micritic limestone } & 0.0 & 0.0 & 0.0 & 0.0 & 0.0 & 0.0 & 0.0 & 0.0 & 0.0 & 0.0 & 0.0 & 0.0 & 0.0\end{array}$

$\begin{array}{llrrrrrrrrrrrrrrrrrr}\text { Sc } & \text { Sparitic limestone } & 0.0 & 1 & 0.2 & 2 & 0.3 & 3 & 0.8 & 3 & 0.7 & 4 & 1.0 & 0.0 & 0.0 & 0.0 & 0.0 & 0.0 & 0.0 & 0.0 \\ \text { Md } & \text { Dolomicrite } & 0.0 & 13 & 3.2 & 16 & 2.6 & 17 & 4.4 & 11 & 2.7 & 2 & 0.5 & 0.0 & 0.0 & 0.0 & 0.0 & 0.0 & 0.0 & 0.0\end{array}$

$\begin{array}{llllllllllllllllllllll}\mathrm{Sd} & \text { Dolosparite } & 7 & 1.7 & 2 & 0.5 & 2 & 0.3 & 3 & 0.8 & 1 & 0.2 & 2 & 0.5 & 0.0 & 7 & 1.6 & 0.0 & 0.0 & 0.0 & 0.0 & 0.0\end{array}$

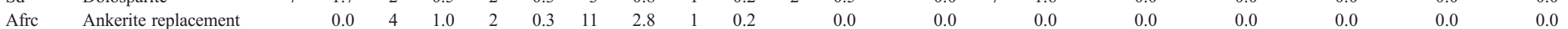

on carbonate

rock fragment

Fo

Ep Echinoderm plates

NCIn Intraclast

CI Mli Micritic limestone TOTAL

0.0

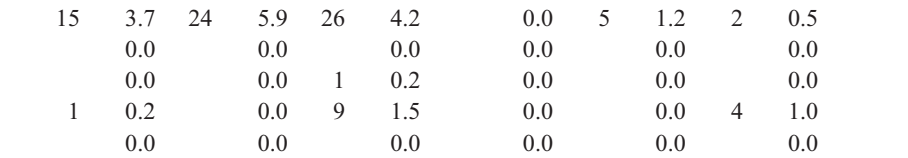

$\begin{array}{lllllll}0.0 & 0.0 & 0.0 & 0.0 & 0.0 & 0.0 & 0.0 \\ 0.0 & 0.0 & 0.0 & 0.0 & 0.0 & 0.0\end{array}$

[Ca] Calcite cement

[Dol] Dolomite cement

C [Anq] Anquerite cement

E [Q] Quartz cement

$\mathrm{M}$ Ank[Q] Anquerite replacing quartz cement

$\begin{array}{llllllllllllllllllllll}349 & 86.8 & 298 & 73.8 & 416 & 67.2 & 332 & 85.3 & 354 & 87.8 & 328 & 82.0 & 313 & 77.7 & 364 & 0.0 & 85.6 & 369 & 0.0 & 0.0 & 0.0 & 0.0 \\ 0.2 & 323 & 85.2 & 340 & 84.4 & 408 & 93.8 & 388 & 94.2\end{array}$

$\begin{array}{lllllllllllllllll}0.0 & 0.0 & 0.0 & 0.0 & 5 & 1.2 & 7 & 1.8 & 0.0 & 3 & 0.7 & 0.0 & 0.0 & 0.0 & 0.0 & 0.0\end{array}$

$\begin{array}{llllllllllllllllllllll}10 & 2.5 & 7 & 1.7 & 3 & 0.5 & 15 & 3.9 & 4 & 1.0 & 4 & 1.0 & 0.0 & 11 & 2.6 & 0.0 & 1 & 0.3 & 0.0 & 0.0 & 0.0\end{array}$

$\begin{array}{rrrrrrrrrrrrrrrrrrrrrrrrrrr}8 & 2.0 & 34 & 8.4 & 75 & 12.1 & 9 & 2.3 & 3 & 0.7 & 8 & 2.0 & & 0.0 & 4 & 0.9 & 2 & 0.5 & 11 & 2.9 & & 0.0 & 0.0 & 0.0 \\ 10 & 2.5 & 11 & 2.7 & 13 & 2.1 & 25 & 6.4 & 28 & 6.9 & 44 & 11.0 & 41 & 10.2 & 26 & 6.1 & 29 & 7.1 & 7 & 1.8 & 47 & 11.7 & 22 & 5.1 & 2 & 0.5\end{array}$

E [Cao] Kaolinite cement

$\mathrm{N}$ Anq[Cao] Anquerite replacing

kaolinite cement

$\begin{array}{llllllllllllllllll}\mathrm{T} & \text { [Cao-il] } & \text { Kaolinite-illite cement } & 1 & 0.2 & 0.0 & 0.0 & 0.0 & 0.0 & 0.0 & 0.0 & 0.0 & 2 & 0.5 & 0.0 & 0.0 & 0.0 & 0.0\end{array}$

$\begin{array}{llllllllllllllll}\mathrm{S} & \text { [il] } \quad \text { Illite cement } & 0.0 & 0.0 & 0.0 & 0.0 & 0.0 & 0.0 & 0.0 & 0.0 & 0.0 & 0.0 & 1 & 0.2 & 0.0 & 0.0\end{array}$

$\begin{array}{llrlllllllllllllllllllllllll}{[\mathrm{Fe}]} & \text { Fe-oxide cement } & 16 & 4.0 & 51 & 12.6 & 101 & 16.3 & 4 & 1.0 & 6 & 1.5 & 1 & 0.3 & 48 & 11.9 & 12 & 2.8 & 6 & 1.5 & 37 & 9.8 & 2 & 0.5 & 5 & 1.1 & 22 & 5.3\end{array}$

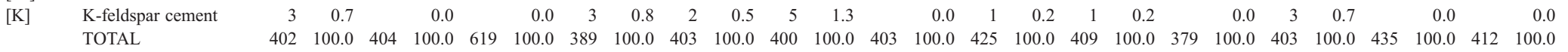

Quartz typologies have been classified following the criteria of Basu et al. (1975). Plutonic rock fragments have been counted as quartz, feldspar and muscovite in plutonic rock fragments (Qfrg, Kfrg, Mfrg). 
can be observed. Magaña Fm. 2 represents more distal deposits of the meandering fluvial system.

Sierra de Matute Fm (DS 2). This formation, $145 \mathrm{~m}$ thick, is only represented in the southeastern section (ALM) (Fig. 3). It is composed of tabular carbonate beds of grey wackestone containing ostracods and charophytes, subordinate gastropods, bivalve fragments and scarce oncolites. Root marks and brecciation related to watertable fluctuations are common. Limestone bodies are less than 4-5 $\mathrm{m}$ thick and interbedded with channelized sandstone bodies, showing trough cross-bedding and carbonate-rock fragments at the base (Fig. 3). The Sierra de Matute Fm. was deposited in a lacustrine-palustrine environment.

\section{Methods}

Samples of medium-grained sandstones were collected from two representative stratigraphic sections of the Tera Group (see Fig. 1 for locations) and 26 selected for analysis. Thin sections were etched and stained using HF and sodium cobaltinitrite for potassium feldspar, and alizarin-red and potassium ferrocyanide for carbonate identification (Chayes, 1952; Lindholm and Finkelman, 1972, respectively). To characterize detrital modes, a quantitative petrographic analysis was performed on thin sections by the integrated Gazzi-Zuffa point counting method (Gazzi, 1966; Zuffa, 1985; Weltje, 2002). This procedure combines the "Gazzi-Dickinson" and traditional criteria (Ingersoll et al., 1984). Four hundred to four hundred and fifty points were counted per slide. Postdepositional modifications to the original framework (e.g. feldspar replacement) were assessed to restore the original composition of the sandstone framework. The petrographic data (Tables 1 and 2) reveal the restored framework compositions, and in each case the way in which composition differs from the original framework is indicated: carbonate replacement of quartz $(\mathrm{Cq})$; kaolinite, kaolinite plus illite, illite or carbonate replacement of K-feldspar (CaoK, Cik, Kill, Ck); illite replacement of albite (Ail); carbonate replacement of plagioclase (Cab); ankerite replacement of carbonate rock fragments (Afrc). Thirty-two detrital classes were considered and grouped into four categories according to the criteria of Zuffa (1980): non-carbonate extrabasinal (NCE), carbonate extrabasinal (CE), non-carbonate intrabasinal (NCI) and carbonate intrabasinal (CI) (Tables 1 and 2).

\section{Results}

Forty-three petrographic classes were recognized, comprising detrital grains (32 classes) and cements (11 classes) (Tables 1 and 2). Depositional matrix composed exclusively of diagenetic phases was not recognized (i.e., pseudomatrix, epimatrix and pore filling). The epimatrix and pseudomatrix affecting the original framework grains were considered, however, when reconstructing the original framework composition (Tables 1 and 2). Diagenetic processes (mechanical and chemical compaction and cementation) were intense and destroyed the porosity of the sediment.

\subsection{Grain types}

\subsubsection{Non-carbonate extrabasinal grains (NCE)}

This category included quartz, K-feldspars, plagioclase, muscovite, dense minerals (pyrite, tourmaline and other opaque minerals), chert, and rock fragments (slate-schist fragments and plutonic rock fragments). Monocrystalline quartz with abraded overgrowths (Qm [Q] in Tables 1 and 2 and Fig. 4B) was observed at the base of both sections; its percentage decreases towards the top of the sections.

Replacement of feldspar by clay minerals and carbonate is common (kaolinite, illite or illite replacement of kaolinite, CaoK, Kil, Cik in Tables 1 and 2). Albites (Ab) (Fig. $4 \mathrm{H}$ ) are commonly replaced by clay minerals (illite) (Ail).

Although scarce, non-carbonate lithic fragments provided important information on source rocks. These included slate and fine-grained schist (Lm) (Fig. 4I), chert grains (Ch) (Fig. 4I) and plutonic rock fragments (Fig. 4F). Chert grains are scarce and either show tectonic fabric with oriented crystal units (of metamorphic origin) (Fig. 4I) or an isotropic texture.

\subsubsection{Carbonate extrabasinal (CE)}

Carbonate-rock fragments were classified according to their composition and grain size as: micritic limestone fragments (Ml) (Fig. 4C), sparitic rock fragments (Sc) (Fig. 4E and $\mathrm{H}$ ), dolomicritic fragments $(\mathrm{Md})$ and dolosparitic fragments (Sd). In addition, recycled fossils of extrabasinal origin (Fo) were also included in this group. Echinoderm plates (Ep) (Fig. 4A) with inherited syntaxial overgrowths (rounded and discontinuous outlines) are common. All these grains were derived from sedimentary units and identified according to the petrographic criteria of Zuffa (1987).

\subsubsection{Noncarbonate intrabasinal (NCI)}

This category is not quantitatively significant and consists of soft silty-clay grains, deformed by mechanical compaction and commonly associated with quartzofeldspathic deposits. 


\subsubsection{Carbonate intrabasinal (CI)}

Composed of micritic limestones associated with calcretes, these grains often show textures of septarian nodules (Fig. 4D).

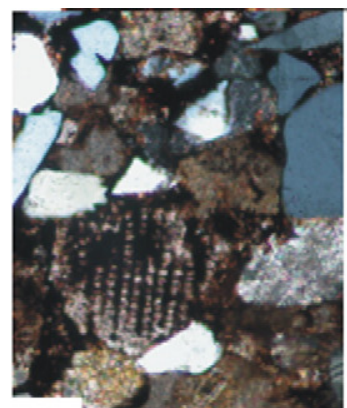

A
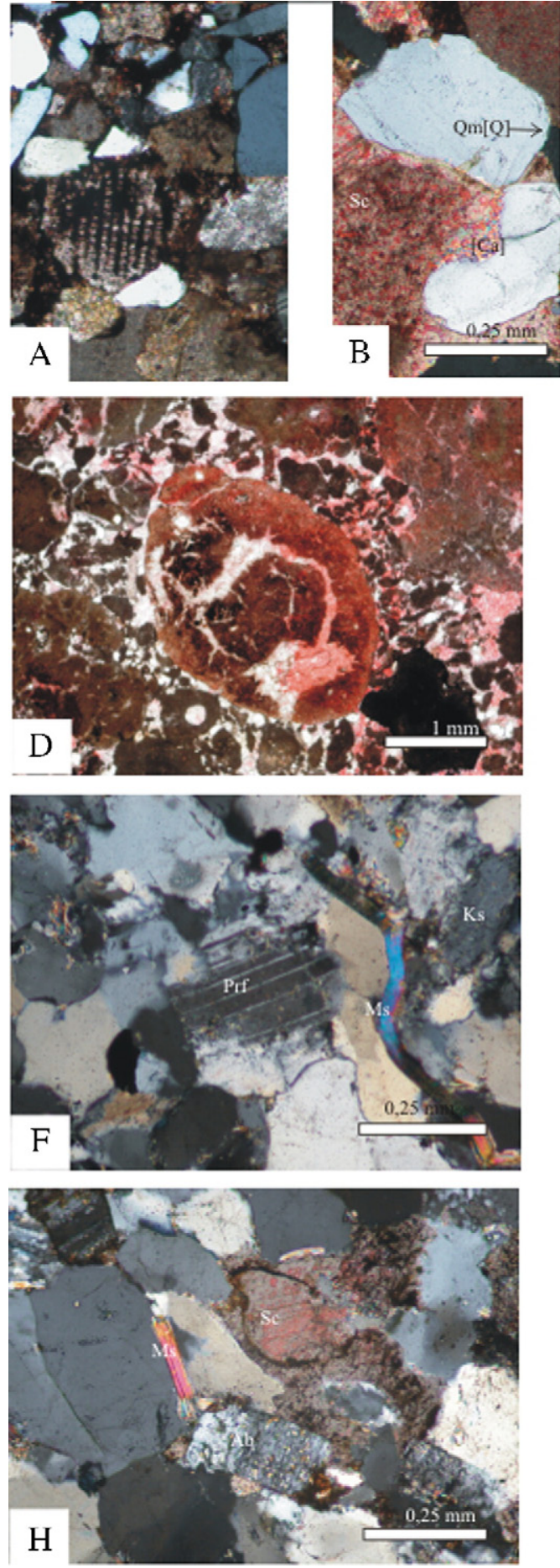

\subsubsection{Recalculated parameters}

For an accurate analysis of clastic modes, we established several petrographic variables. Table 3 summarizes the criteria used to obtain these variables
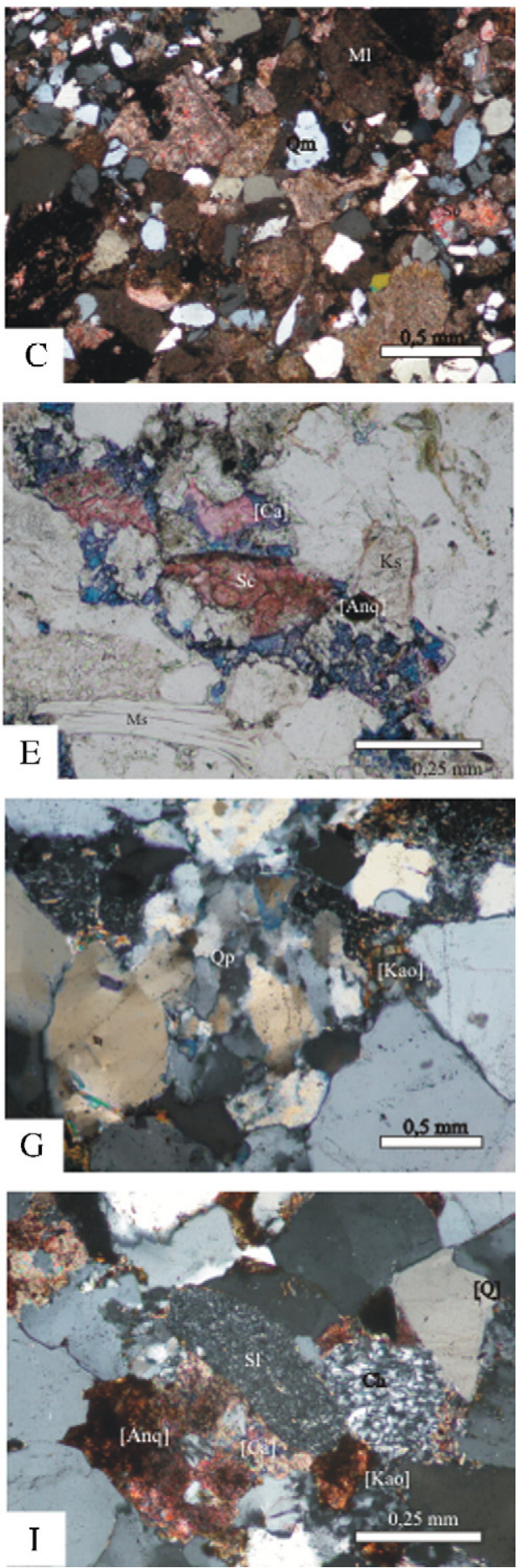
Table 3

Recalculated parameters used in the ternary plots

\begin{tabular}{|c|c|}
\hline Ternary plot & Parámeters \\
\hline NCE-CE-CI & $\begin{array}{l}\mathrm{NCE}=\mathrm{Qmr}+\mathrm{Qmo}+\mathrm{Qm}[\mathrm{Q}]+\mathrm{Qp} 2-3+\mathrm{Qp}>3+\mathrm{Qfrg} \\
+\mathrm{Cq}+\mathrm{Ks}+\mathrm{Kfrg}+\mathrm{CaoK}+\mathrm{Cik}+\mathrm{Cil}+\mathrm{Ck}+\mathrm{Ps}+\mathrm{Ab}+\mathrm{Ail} \\
+\mathrm{Cab}+\mathrm{Ch}+\mathrm{Lm}+\mathrm{Ms}+\mathrm{Mfrg}+\mathrm{Tu}+\mathrm{Opp} \\
\mathrm{CE}=\mathrm{Ml}+\mathrm{Sc}+\mathrm{Md}+\mathrm{Sd}+\mathrm{Afrc}+\mathrm{Fo}+\mathrm{E} \\
\mathrm{CI}=\mathrm{In}\end{array}$ \\
\hline QFR & $\begin{array}{l}Q=\mathrm{Qmr}+\mathrm{Qmo}+\mathrm{Qm}[\mathrm{Q}]+\mathrm{Qp} 2-3+\mathrm{Qp}>3+\mathrm{Qfrg}+\mathrm{Cq} \\
F=\mathrm{Ks}+\mathrm{Kfrg}+\mathrm{CaoK}+\mathrm{Cik}+\mathrm{Kil}+\mathrm{Ck}+\mathrm{Ps}+\mathrm{Ab}+\mathrm{Ail} \\
+\mathrm{Cab} \\
R=\mathrm{Qfrp}+\mathrm{Kfrg}+\mathrm{Mfrg}+\mathrm{Lm}+\mathrm{CE}\end{array}$ \\
\hline QmFLt & $\begin{array}{l}\mathrm{Qm}=\mathrm{Qmr}+\mathrm{Qmo}+\mathrm{Qm}[\mathrm{Q}]+\mathrm{Qp} 2-3+\mathrm{Qp}>3+\mathrm{Qfrg} \\
+\mathrm{Cq} \\
F=\mathrm{Ks}+\mathrm{Kfrg}+\mathrm{CaoK}+\mathrm{Cik}+\mathrm{Kil}+\mathrm{Ck}+\mathrm{Ps}+\mathrm{Ab}+\mathrm{Ail} \\
+\mathrm{Cab} \\
\mathrm{Lt}=\mathrm{Ch}+\mathrm{Lm}+\mathrm{Ml}+\mathrm{Sc}+\mathrm{Md}+\mathrm{Sd}+\mathrm{Afrc}+\mathrm{Fo}+\mathrm{Pe}\end{array}$ \\
\hline QmKP & $\begin{array}{l}\mathrm{Qm}=\mathrm{Qmr}+\mathrm{Qmo}+\mathrm{Qm}[\mathrm{Q}]+\mathrm{Qp} 2-3+\mathrm{Qp}>3+\mathrm{Qfrg} \\
+\mathrm{Cq} \\
K=\mathrm{Ks}+\mathrm{Kfrg}+\mathrm{CaoK}+\mathrm{Cik}++\mathrm{Kil}+\mathrm{Ck} \\
P=\mathrm{Ps}+\mathrm{Ab}+\mathrm{Ail}+\mathrm{Cab}\end{array}$ \\
\hline QmrQmoQp & $\begin{array}{l}\mathrm{Qmr}=\mathrm{Qmr} \\
\mathrm{Qmo}=\mathrm{Qmo} \\
\mathrm{Qp}=\mathrm{Qp} 2-3+\mathrm{Qp}>3\end{array}$ \\
\hline RgRsRm & $\begin{array}{l}\mathrm{Rg}=\mathrm{Qfrg}+\mathrm{Kfrg}+\mathrm{Mfrg} \\
\mathrm{Rs}=\mathrm{CE} \\
\mathrm{Rm}=\mathrm{Lm}\end{array}$ \\
\hline LmLsmLse & $\begin{array}{l}\mathrm{Lm}=\mathrm{Lm} \\
\mathrm{Lsm}=\mathrm{Ml}+\mathrm{Md}+\mathrm{Afrc} \\
\mathrm{Lse}=\mathrm{Sc}+\mathrm{Sd}+\mathrm{Fo}+\mathrm{Pe}\end{array}$ \\
\hline
\end{tabular}

See Tables 1 and 2 for abreviations.

and Table 4 provides the values for both sections. These recalculated parameters were used to plot clastic modes in ternary diagrams (Fig. 4). The Qmr/Qmo and Qp/Qm ratios obtained (Fig. 3) proved to be sensitive indicators of metamorphic source areas. To characterize the depositional sequences we also used Ms/Qmr ratios (Fig. 3).

\subsection{Sandstone composition and petrofacies}

The sandstone framework composition was represented in different ternary plots (Fig. 5) following the criteria of several authors: Zuffa (1985) (NCE/CI/CE); Dickinson et al. (1983) (QmFLt); Dickinson (1985) (QmKP); Basu et al. (1975) (Qmr/Qmo/Qp); Arribas et al. (1990) and Criteri and Le Pera (1994) (RgRsRm); Arribas et al. (2003) (LmLsmLse). Three different petrofacies were distinguished on the basis of these plots. Their signatures and main characteristics are detailed in Table 5, in the form of arithmetic averages and standard deviations for all recalculated parameters. Rigorous statistical analyses were not attempted in our reconnaissance study of the provenance of the Tera Group in the Eastern part of Cameros Basin.

Petrofacies 1: This sedimentolithic petrofacies plots in the "recycled orogenic" field of the QmFLt diagram (Fig. 5B). It shows a high lithic fragment content, mainly including micritic carbonate fragments (Fig. 4C), and plots on the Rs pole of the RgRsRm ternary diagram and on the Lsm pole of the LmLsmLse diagram (Fig. 5E and F). Fragments from old carbonate cements or recrystalized fossils ascribed to marine carbonate facies were observed. Inherited echinoderm plates (Fig. 5A) with syntaxial cements are also present, and there is evidence of intrabasinal contributions from calcretes (Fig. 4D). The Qmr/Qmo ratio is high (Table 4, Fig. 3), and some quartz grains display abraded overgrowths. Feldspars and polycrystalline quartz are also rare (see Qp/Qm Table 4, Fig. 3). The Ágreda Fm. (DS 1) is characterized by Petrofacies 1 .

Petrofacies 2: Petrofacies 2 is quarzofeldspathic and plots within the "craton interior" field of the QmFLt ternary plot (Fig. 5B). Plutonic rock fragments and schist-slate fragments are present. Polycrystalline quartz, usually with a tectonic fabric (Fig. 4G) and more than three crystal units, is common. Relatively high K-feldspar and muscovite contents are characteristic of this petrofacies, tending to increase towards the top (Fig. 5C). Petrofacies 2 was formed during the deposition of the Magaña Fm. (DS 2).

This petrofacies is not compositionally homogeneous in the southeastern and northwestern sections of the

Fig. 4. Photomicrographs of the detrital components and diagenetic features of the Tera Group sandstones. A.- - Inherited echinoderm plate (Ep) (ALM, Petrofacies 1, Ágreda Fm.). Crossed nichols. B.-Monocrystalline quartz grain with inherited syntaxial overgrowth (Qm[Q]). (ALM, Petrofacies 1, Ágreda Fm.). Crossed nichols. C.—Litharenite from the Ágreda Fm., Petrofacies 1 (ALM). Note the high content of monocrystalline quartz $(\mathrm{Qm})$ and micritic limestone fragments (Ml). Crossed nichols. D.- -Septarian nodule of a calcrete from the Ágreda Fm. (Petrofacies 1). Crossed nichols. E.-Medium-grained arkose from the Magaña Fm. (MOV, Petrofacies 2B) showing a sparitic rock fragment (Sc). A ferroan calcite cement ([Ca]) and an ankerite cement ([Anq]) also appear. Parallel nichols. F.-Medium-grained arkose from the Magaña Fm. (MOV, Petrofacies 2B) with a plutonic rock fragment (Prf), moscovite (Ms) and single grains of K-feldspar (Ks). The framework exhibits dense packing generated by compaction. Crossed nichols. G.-Petrofacies 2A (ALM, Ágreda Fm.) containing polycrystalline quartz (Qp) with tectonic fabric. Kaolinite pore filling ([Kao]) is very common in this petrofacies. Crossed nichols. H.- Subarkose from the Sierra de Matute Fm. (ALM, Petrofacies 3) showing a sparitic rock fragment $(\mathrm{Sc})$. Note the presence of moscovite $(\mathrm{Ms})$ and albite $(\mathrm{Ab})$. Crossed nichols. I.- Subarkose from the Sierra de Matute Fm. (ALM, Petrofacies 3) with slate (Sl) and chert (Ch) fragments. As diagenetic features, it shows quartz overgrowths ([Q]), ankerite overgrowths ([Anq]), calcite overgrowths ([Ca]) and kaolinite pore fillings ([Kao]). Crossed nichols. 
Table 4

Values of recalculated parameters for sandstones of Almajano (ALM) and Montenegro-Villoslada (MOV) sections (Arithmetic averages)

\begin{tabular}{|c|c|c|c|c|c|c|c|c|c|c|c|c|c|c|c|c|c|c|c|c|c|c|c|c|c|c|}
\hline Samples & $\begin{array}{l}\text { ALM- } \\
101\end{array}$ & $\begin{array}{l}\text { ALM- } \\
102\end{array}$ & $\begin{array}{l}\text { ALM- } \\
103\end{array}$ & $\begin{array}{l}\text { ALM- } \\
104\end{array}$ & $\begin{array}{l}\text { ALM- } \\
105\end{array}$ & $\begin{array}{l}\text { ALM- } \\
106\end{array}$ & $\begin{array}{l}\text { ALM- } \\
107\end{array}$ & $\begin{array}{l}\text { ALM- } \\
108\end{array}$ & $\begin{array}{l}\text { ALM- } \\
109\end{array}$ & $\begin{array}{l}\text { ALM- } \\
110\end{array}$ & $\begin{array}{l}\text { ALM- } \\
111\end{array}$ & $\begin{array}{l}\text { ALM- } \\
112\end{array}$ & $\begin{array}{l}\text { ALM- } \\
113\end{array}$ & $\begin{array}{l}\text { MOV- } \\
100\end{array}$ & $\begin{array}{l}\text { MOV- } \\
101\end{array}$ & $\begin{array}{l}\text { MOV- } \\
102\end{array}$ & $\begin{array}{l}\text { MOV- } \\
103\end{array}$ & $\begin{array}{l}\text { MOV- } \\
104\end{array}$ & $\begin{array}{l}\text { MOV- } \\
105\end{array}$ & $\begin{array}{l}\text { MOV- } \\
106\end{array}$ & $\begin{array}{l}\text { MOV- } \\
107\end{array}$ & $\begin{array}{l}\text { MOV- } \\
108\end{array}$ & $\begin{array}{l}\text { MOV- } \\
109\end{array}$ & $\begin{array}{l}\text { MOV- } \\
110\end{array}$ & $\begin{array}{l}\text { MOV- } \\
111\end{array}$ & $\begin{array}{l}\text { MOr } \\
112\end{array}$ \\
\hline NCE $\%$ & 43.6 & 50.9 & 6.4 & 49.9 & 83.1 & 100.0 & 100.0 & 100.0 & 100.0 & 100.0 & 90.2 & 83.9 & 80.8 & 93.4 & 85.2 & 86.1 & 89.8 & 94.1 & 95.7 & 100.0 & 98.1 & 100.0 & 99.4 & 100.0 & 100.0 & 100.0 \\
\hline $\mathrm{CE} \%$ & 56.0 & 48.6 & 32.9 & 14.6 & 16.9 & 0.0 & 0.0 & 0.0 & 0.0 & 0.0 & 9.8 & 16.1 & 19.2 & 6.3 & 14.8 & 11.8 & 10.2 & 5.9 & 3.0 & 0.0 & 1.9 & 0.0 & 0.0 & 0.0 & 0.0 & 0.0 \\
\hline $\mathrm{CI} \%$ & 0.4 & 0.6 & 60.7 & 35.5 & 0.0 & 0.0 & 0.0 & 0.0 & 0.0 & 0.0 & 0.0 & 0.0 & 0.0 & 0.3 & 0.0 & 2.2 & 0.0 & 0.0 & 1.2 & 0.0 & 0.0 & 0.0 & 0.6 & 0.0 & 0.0 & 0.0 \\
\hline $\mathrm{Qm} \%$ & 43.9 & 45.2 & 16.4 & 73.5 & 77.7 & 82.7 & 86.4 & 82.4 & 80.2 & 86.5 & 74.1 & 64.4 & 61.4 & 69.3 & 66.7 & 65.1 & 68.5 & 71.1 & 71.8 & 70.3 & 66.8 & 67.5 & 84.1 & 70.1 & 80.4 & 65.0 \\
\hline $\mathrm{F} \%$ & 1.5 & 3.9 & 0.0 & 3.5 & 4.5 & 15.9 & 13.0 & 17.1 & 18.3 & 12.9 & 13.7 & 17.4 & 16.8 & 22.4 & 21.4 & 21.9 & 18.2 & 21.8 & 22.8 & 23.3 & 27.7 & 28.3 & 15.1 & 27.8 & 16.7 & 33.1 \\
\hline $\mathrm{Lt} \%$ & 54.6 & 50.9 & 83.6 & 23.0 & 17.8 & 1.4 & 0.6 & 0.6 & 1.4 & 0.6 & 12.2 & 18.1 & 21.8 & 8.3 & 11.9 & 13.0 & 13.4 & 7.2 & 5.4 & 6.4 & 5.5 & 4.2 & 0.8 & 2.1 & 2.9 & 1.8 \\
\hline $\mathrm{Qm} \%$ & 43.9 & 45.2 & 16.4 & 73.5 & 77.7 & 82.7 & 86.4 & 82.4 & 80.2 & 86.5 & 74.1 & 64.4 & 61.4 & 69.3 & 66.7 & 65.1 & 68.5 & 71.1 & 71.8 & 70.3 & 66.8 & 67.5 & 84.1 & 70.1 & 80.4 & 65.0 \\
\hline $\mathrm{K} \%$ & 1.5 & 3.9 & 0.0 & 3.5 & 4.5 & 15.9 & 13.0 & 17.1 & 18.3 & 12.9 & 13.7 & 17.4 & 16.8 & 22.4 & 21.4 & 21.9 & 18.2 & 21.8 & 22.8 & 23.3 & 27.7 & 28.3 & 15.1 & 27.8 & 16.7 & 33.1 \\
\hline $\mathrm{P} \%$ & 54.6 & 50.9 & 83.6 & 23.0 & 17.8 & 1.4 & 0.6 & 0.6 & 1.4 & 0.6 & 12.2 & 18.1 & 21.8 & 8.3 & 11.9 & 13.0 & 13.4 & 7.2 & 5.4 & 6.4 & 5.5 & 4.2 & 0.8 & 2.1 & 2.9 & 1.8 \\
\hline Qmr $\%$ & 43.9 & 45.2 & 44.4 & 73.5 & 77.7 & 82.7 & 86.4 & 82.4 & 80.2 & 86.5 & 74.1 & 64.4 & 61.4 & 69.3 & 66.7 & 65.1 & 68.5 & 71.1 & 71.8 & 70.3 & 66.8 & 67.5 & 84.1 & 70.1 & 80.4 & 65.0 \\
\hline Qmo\% & 1.5 & 3.9 & 55.6 & 3.5 & 4.5 & 15.9 & 13.0 & 17.1 & 18.3 & 12.9 & 13.7 & 17.4 & 16.8 & 22.4 & 21.4 & 21.9 & 18.2 & 21.8 & 22.8 & 23.3 & 27.7 & 28.3 & 15.1 & 27.8 & 16.7 & 33.1 \\
\hline $\mathrm{Qp} \%$ & 54.6 & 50.9 & 0.0 & 23.0 & 17.8 & 1.4 & 0.6 & 0.6 & 1.4 & 0.6 & 12.2 & 18.1 & 21.8 & 8.3 & 11.9 & 13.0 & 13.4 & 7.2 & 5.4 & 6.4 & 5.5 & 4.2 & 0.8 & 2.1 & 2.9 & 1.8 \\
\hline $\mathrm{Rg} \%$ & 0.0 & 0.0 & 0.0 & 0.0 & 0.0 & 82.7 & 86.4 & 82.4 & 80.2 & 86.5 & 0.0 & 0.0 & 0.0 & 0.0 & 0.0 & 0.0 & 0.0 & 0.0 & 0.0 & 0.0 & 0.0 & 67.5 & 0.0 & 70.1 & 80.4 & 65.0 \\
\hline Rs $\%$ & 1.5 & 3.9 & 55.6 & 3.5 & 4.5 & 0.0 & 0.0 & 0.0 & 0.0 & 0.0 & 13.7 & 17.4 & 16.8 & 22.4 & 21.4 & 21.9 & 18.2 & 21.8 & 22.8 & 0.0 & 27.7 & 0.0 & 0.0 & 0.0 & 0.0 & 0.0 \\
\hline $\mathrm{Rm} \%$ & 0.0 & 0.0 & 0.0 & 0.0 & 17.8 & 1.4 & 0.0 & 0.0 & 0.0 & 0.0 & 12.2 & 18.1 & 21.8 & 8.3 & 11.9 & 13.0 & 13.4 & 7.2 & 5.4 & 6.4 & 5.5 & 4.2 & 0.0 & 2.1 & 2.9 & 0.0 \\
\hline $\mathrm{Lm} \%$ & 0.0 & 0.0 & 0.0 & 0.0 & 0.0 & 100.0 & 0.0 & 0.0 & 0.0 & 0.0 & 2.5 & 1.4 & 2.4 & 50.0 & 9.1 & 8.0 & 8.1 & 11.1 & 11.1 & 100.0 & 36.4 & 100.0 & 0.0 & 100.0 & 100.0 & 0.0 \\
\hline Lsm\% & 68.5 & 81.1 & 91.3 & 83.1 & 52.9 & 0.0 & 0.0 & 0.0 & 0.0 & 0.0 & 2.5 & 5.5 & 19.3 & 50.0 & 13.6 & 16.0 & 16.2 & 22.2 & 66.7 & 0.0 & 63.6 & 0.0 & 0.0 & 0.0 & 0.0 & 0.0 \\
\hline Lse $\%$ & 31.5 & 18.9 & 8.7 & 16.9 & 45.1 & 0.0 & 0.0 & 0.0 & 0.0 & 0.0 & 95.0 & 93.2 & 78.3 & 0.0 & 77.3 & 76.0 & 75.7 & 66.7 & 22.2 & 0.0 & 0.0 & 0.0 & 0.0 & 0.0 & 0.0 & 0.0 \\
\hline$P / F$ & 0.2 & 1.7 & & 0.6 & 0.6 & 5.0 & 0.5 & 0.5 & & & 5.9 & 11.8 & 9.6 & 7.0 & 9.5 & 5.9 & 7.0 & 7.2 & 2.0 & 4.2 & 4.0 & 3.9 & 5.5 & 3.0 & 2.5 & 5.1 \\
\hline $\begin{array}{l}\text { Qmr/ } \\
\text { Qmo }\end{array}$ & 0.6 & 0.3 & 0.8 & 0.6 & 0.1 & 0.1 & 0.1 & 0.1 & 0.0 & 0.0 & 0.1 & 0.0 & 0.2 & 0.4 & 0.2 & 0.3 & 0.2 & 0.5 & 0.2 & 0.0 & 0.2 & 0.1 & 0.1 & 0.0 & 0.1 & 0.1 \\
\hline $\mathrm{Qp} / \mathrm{Qm}$ & 0.1 & 0.0 & 0.0 & 0.3 & 0.3 & 0.4 & 0.2 & 0.4 & 0.3 & 0.5 & 0.3 & 0.6 & 0.4 & 0.4 & 0.2 & 0.2 & 0.3 & 0.6 & 0.3 & 0.1 & 0.3 & 0.3 & 0.1 & 0.3 & 0.3 & 0.3 \\
\hline $\begin{array}{l}\text { Mosc/ } \\
\text { Qmr }\end{array}$ & 0.0 & 0.0 & 0.0 & 0.0 & 0.1 & 0.5 & 0.2 & 0.9 & 0.7 & 0.6 & 0.4 & 1.0 & 0.9 & 0.6 & 0.8 & 1.0 & 0.1 & 0.0 & 0.2 & 2.1 & 0.7 & 0.7 & 6.3 & 2.0 & 5.5 & 6.0 \\
\hline
\end{tabular}



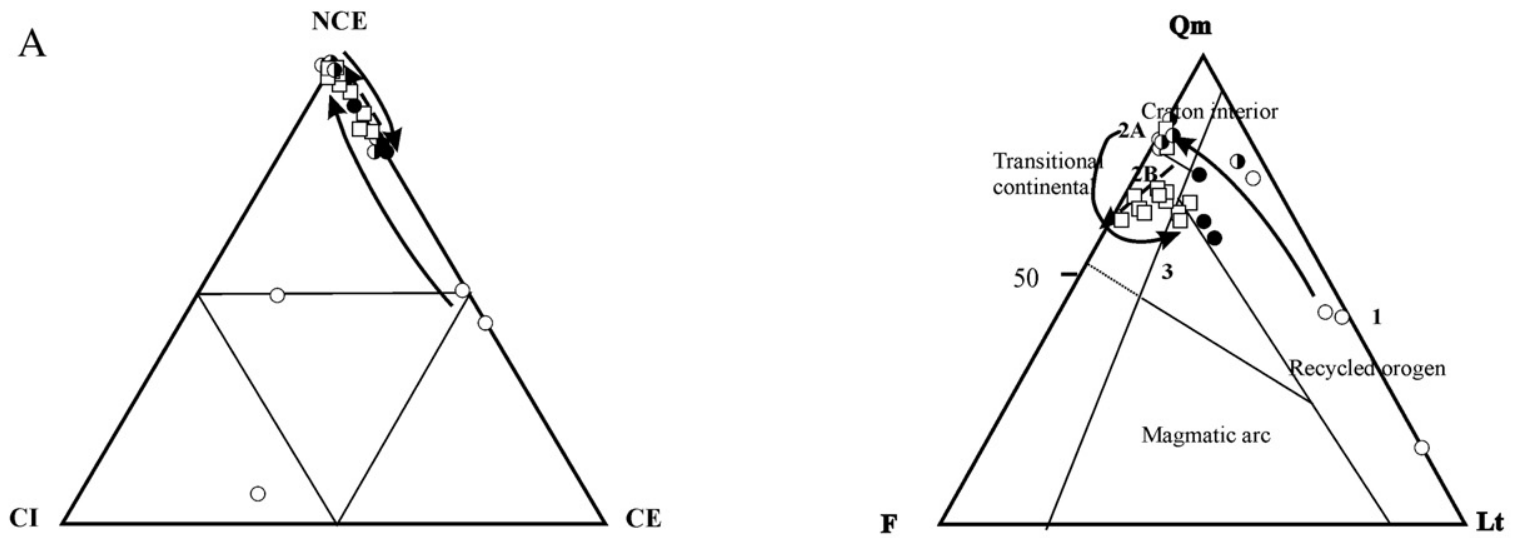

B
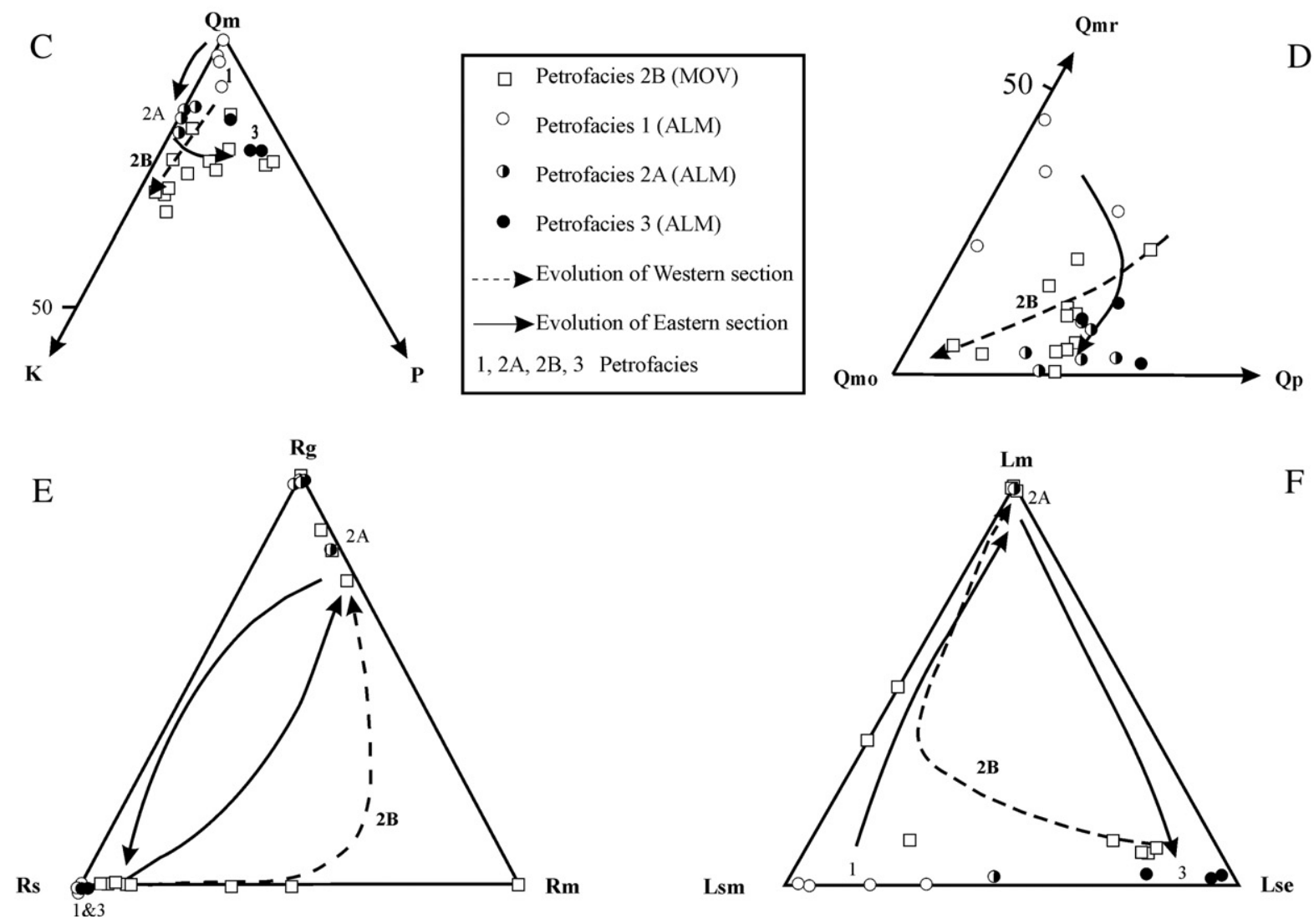

Fig. 5. Ternary plots describing sandstone composition. Values for the northwestern and the southeastern sections are shown as squares and circles, respectively (see legend). Development for the western section (MOV) is marked by broken arrows; development for the southeastern section (ALM) is indicated by continuous arrows. Petrofacies are indicated with numbers when possible. See Table 3 for recalculated parameters and Tables 1 and 2 for numerical values. The ternary diagrams were prepared according to the criteria of several authors: A. - Zuffa (1985) (NCE/CI/CE); B.Dickinson et al. (1983) (QmFLt); C.—Dickinson (1985) (QmKP); D.—Basu et al. (1975) (Qmr/Qmo/Qp); E. - Arribas et al. (1990) and Criteri and Le Pera (1994) (RgRsRm); F.-Arribas et al. (2003) (LmLsmLse).

basin. Thus, it is possible to distinguish Petrofacies 2A from $2 \mathrm{~B}$. Petrofacies $2 \mathrm{~A}$ is represented in the "craton interior" field of the QmFLt ternary plot, whereas
Petrofacies 2B evolves from the "craton interior" to the "transitional continental" field of the QmFLt ternary plot (Fig. 5B). Petrofacies 2A plots along the Rg-Rm 
Table 5

Main characteristics of the three petrofacies. $\left(^{*}\right)$ means arithmetic averages and standard deviations

\begin{tabular}{|c|c|c|c|c|c|c|c|c|}
\hline Petrofacies & Samples & Lithology & QmFLt* & QmKP* & $\mathrm{Qp} / \mathrm{Qm} *$ & Qmr/Qmo* & $\mathrm{P} / \mathrm{K}^{*}$ & $\begin{array}{l}\text { Main characteristics and } \\
\text { Lithostratigraphic units }\end{array}$ \\
\hline 1: Sedimentolithic & $\begin{array}{l}\text { ALM-101 } \\
\text { ALM-102 } \\
\text { ALM-103 } \\
\text { ALM-104 }\end{array}$ & Sedarenites & $\begin{array}{l}\mathrm{Qm}_{54 \pm 23} \\
\mathrm{~F}_{3 \pm 2} \\
\mathrm{Lt}_{43 \pm 25}\end{array}$ & $\begin{array}{l}\mathrm{Qm}_{45 \pm 23} \\
\mathrm{~K}_{2 \pm 2} \\
\mathrm{P}_{53 \pm 25}\end{array}$ & $0.08 \pm 0.12$ & $0.54 \pm 0.20$ & $0.85 \pm 0.78$ & $\begin{array}{l}\text { Lithic fragments } \\
\text { Micritic carbonate fragments } \\
\text { Inherited echinoderm plates } \\
\text { Intrabasinal contributions } \\
\text { from calcretes } \\
\text { Ágreda Fm. (ALM) }\end{array}$ \\
\hline 2A: Quarzofeldspathic & $\begin{array}{l}\text { ALM-105 } \\
\text { ALM-106 } \\
\text { ALM-107 } \\
\text { ALM-108 } \\
\text { ALM-109 } \\
\text { ALM-110 }\end{array}$ & Mainly subarkoses & $\begin{array}{l}\mathrm{Qm}_{84 \pm 4} \\
\mathrm{~F}_{15 \pm 5} \\
\mathrm{Lt}_{1 \pm 7}\end{array}$ & $\begin{array}{l}\mathrm{Qm}_{85 \pm 3} \\
\mathrm{~K}_{13 \pm 5} \\
\mathrm{P}_{2 \pm 7}\end{array}$ & $0.35 \pm 0.09$ & $0.07 \pm 0.20$ & $1.65 \pm 2.22$ & $\begin{array}{l}\text { Plutonic rock fragments } \\
\text { Squist-slate fragments } \\
\text { Qp }>3 \text { with tectonic fabric } \\
\text { K-feldspar } \\
\text { Muscovite } \\
\text { Magaña Fm. (ALM) }\end{array}$ \\
\hline 2B: Quarzofeldspathic & $\begin{array}{l}\text { MOV-100 MOV-107 } \\
\text { MOV-101 MOV-108 } \\
\text { MOV-102 MOV-109 } \\
\text { MOV-103 MOV-110 } \\
\text { MOV-104 MOV-111 } \\
\text { MOV-105 MOV-112 } \\
\text { MOV-106 MOV-113 }\end{array}$ & Mainly subarkoses & $\begin{array}{l}\mathrm{Qm}_{71 \pm 6} \\
\mathrm{~F}_{23 \pm 5} \\
\mathrm{Lt}_{6 \pm 4}\end{array}$ & $\begin{array}{l}\mathrm{Qm}_{71 \pm 6} \\
\mathrm{~K}_{23 \pm 5} \\
\mathrm{P}_{6 \pm 4}\end{array}$ & $0.70 \pm 0.14$ & $\begin{array}{l}0.27 \pm 0.16 \\
\text { (Magaña Fm. 1) } \\
0.08 \pm 0.05 \\
\text { (Magaña Fm. 2) }\end{array}$ & $5.14 \pm 2.98$ & $\begin{array}{l}\text { Carbonate rock } \\
\text { fragments at the botton } \\
\text { Plutonic rock fragments } \\
\text { at the top } \\
\text { Squist-slate fragments } \\
\text { Qp }>3 \text { with tectonic fabric } \\
\text { K-feldspar } \\
\text { Muscovite } \\
\text { Magaña Fm. } 1 \text { and } 2 \\
\text { (MOV) }\end{array}$ \\
\hline $\begin{array}{l}\text { 3: Quarzo- } \\
\text { sedimentolithic }\end{array}$ & $\begin{array}{l}\text { ALM-111 } \\
\text { ALM-112 } \\
\text { ALM-113 }\end{array}$ & $\begin{array}{l}\text { Subarkoses and } \\
\text { lithoarenites }\end{array}$ & $\begin{array}{l}\mathrm{Qm}_{67 \pm 7} \\
\mathrm{~F}_{16 \pm 2} \\
\mathrm{Lt}_{17 \pm 5}\end{array}$ & $\begin{array}{l}\mathrm{Qm}_{67 \pm 7} \\
\mathrm{~K}_{16 \pm 2} \\
\mathrm{P}_{17 \pm 5}\end{array}$ & $0.44 \pm 0.12$ & $0.13 \pm 0.20$ & $9.11 \pm 2.14$ & $\begin{array}{l}\text { Sparitic rock fragments } \\
\text { Inherited echinoderm plates } \\
\text { Scarce schist-slate fragments } \\
\text { Qp }>3 \text { with tectonic fabric } \\
\text { Sierra de Matute Fm. (ALM) }\end{array}$ \\
\hline
\end{tabular}


edge of the RgRsRm diagram (Fig. 5E). Petrofacies 2B records an evolution from the Rs pole to $\mathrm{Rg}-\mathrm{Rm}$ edge of the ternary plot. Petrofacies 2A shows lower Qmr/Qmo, $\mathrm{Qp} / \mathrm{Qm}$ and $\mathrm{P} / \mathrm{K}$ indices than Petrofacies 2B (Table 4, Figs. 3 and $5 \mathrm{C}$ ). Petrofacies $2 \mathrm{~A}$ shows rare lithic fragments (Fig. 5B). Carbonate-rock fragments (Fig. 4E) are present at the base of Petrofacies 2B (Fig. 4E).

Petrofacies 2B also suggests compositional differences between Magaña Fm. 1 and Magaña Fm. 2, since the Ms/Qmr ratio is higher for Magaña Fm. 2 than Fm. 1 (Fig. 3). In addition, the Qmr/Qmo ratio is higher for Magaña Fm. 1 than for Magaña Fm. 2 (Table 4, Fig. 3).

Petrofacies 3: This quartzolithic petrofacies plots in the "recycled orogenic" field of the QmFLt ternary plot, as does Petrofacies 1 (Fig. 5B). The main petrographic characteristic is the presence of sparitic rock fragments (Fig. 4H) and inherited echinoderm plates. This petrofacies plots close to the Rs pole of the RgRsRm ternary diagram and close to the Lse pole of the LmLsmLse ternary diagram (Fig. 5E and F). Scarce slate-schist fragments occur (Fig. 4I). Other petrographic features are a low Qmr/Qmo ratio (Table 4, Fig. 3), abundant polycrystalline oriented quartz with more than three crystal units (see Qp/Qm in Table 4), and a P/K index higher than that for Petrofacies 2 (Table 4). Petrofacies 3 was generated during the deposition of the Sierra de Matute Fm. (DS 2).

\section{Discussion}

The changes observed in the petrofacies examined in this study indicate variations in source areas during sedimentation of the Tera Group.

Most of the micritic carbonate fragments of Petrofacies 1 are intrabasinal, and are probably derived from coeval calcretes of the Ágreda Fm. Sparitic rock fragments in Petrofacies 1 were supplied by the Jurassic sedimentary substratum. Petrofacies 2 shows an increase in metamorphic and secondary plutonic supplies, recorded in the RgRsRm and LmLsmLse ternary plots (Fig. 5E and F).

The Qmr/Qmo/Qp ternary plot and the Qp/Qm and Qmr/Qmo ratios (Figs. 5D and 3, Table 4) indicate a decrease in non-undulatory monocrystalline quartz and increased polycrystalline typologies at the top of both sections. Polycrystalline quartz with tectonic fabric, indicative of metamorphic sources, is also common at the top.

The three established petrofacies are clearly related to the lithostratigraphic units studied and record the sedimentary evolution of the Tera Group. The Ágreda Fm. (Petrofacies 1) is a recycled (sedimentoclastic) unit, as revealed by its high content of carbonate fragments, inherited echinoderm plates, its low feldspar content and dominance of monocrystalline quartz types (Table 4 ) and recycled patterns. Petrofacies 1 records the erosion of the Jurassic marine pre-rift deposits, mainly the Kimmeridgian limestones of the underlying unit (Torrecilla en Cameros Fm.). This conclusion is supported by the similarity of the extrabasinal carbonate-rock fragments to the Torrecilla en Cameros Fm. limestones (Alonso and Mas, 1990; Benito, 2001; Benito and Mas, 2001, 2002). However, the high Qmr/Qmo index and the presence of quartz with inherited syntaxial overgrowths suggest that Petrofacies 1 also records the erosion of pre-rift Mesozoic siliciclastic units (Callovian quartz-arenites, Mas et al., 2003).

Petrofacies 1 corresponds to the undissected-transitional stage of the non-volcanic rifted margin of Garzanti et al. (2001, 2003). This type of provenance has been recognized in present-day sands of the Red Sea and Gulf of Aden (Yemen). Petrofacies 1 shows many features of this stage: it plots in the same compositional area of the QmFLt ternary plot and shows sedimentary detritus, including recycled monocrystalline quartz and carbonate grains.

Petrofacies 1 is equivalent to Petrofacies A from the western sector of the Cameros Basin (Arribas et al., 2003). Two main differences exist between both petrofacies: (1) Petrofacies 1 is less quartzose than Petrofacies A, which is richer in carbonate lithic fragments (Petrofacies 1: $\mathrm{Qm}_{54} \mathrm{~F}_{3} \mathrm{Lt}_{43}$; Petrofacies A: $\left.\mathrm{Qm}_{85} \mathrm{~F}_{2} \mathrm{Lt}_{13}\right)$; and (2): Petrofacies 1 contains no metamorphic rock fragments, unlike Petrofacies A. Thus, sedimentary sources seem to be more important in Petrofacies 1, which does not record erosion of the crystalline basement. Sandstones in the eastern sector of Cameros Basin have a signature that is closer to the undissected-transitional stage of Garzanti et al. (2001, 2003).

A complex provenance from metamorphic and granite terranes can be deduced for Petrofacies 2 (Magaña Fm., DS 2). Despite the low percentage of slate-schist fragments $(<2 \%)$, the presence of these rock fragments is highly significant. Several studies on modern sediments (Palomares and Arribas, 1993; Arribas and Tortosa, 2003; Le Pera and Arribas, 2004) have demonstrated that sandstone composition does not allow direct quantitative estimations of the lithologies at the source, owing to the different capacities of rocks to generate sands (Palomares and Arribas, 1993). Indeed, slates and schists show a very low sand-generating capacity (Sand Generation Index, Palomares and Arribas, 1993) and are often underrepresented, because of their low rock-fragment content. Slateschist fragments are therefore considered significant despite their scarcity. 


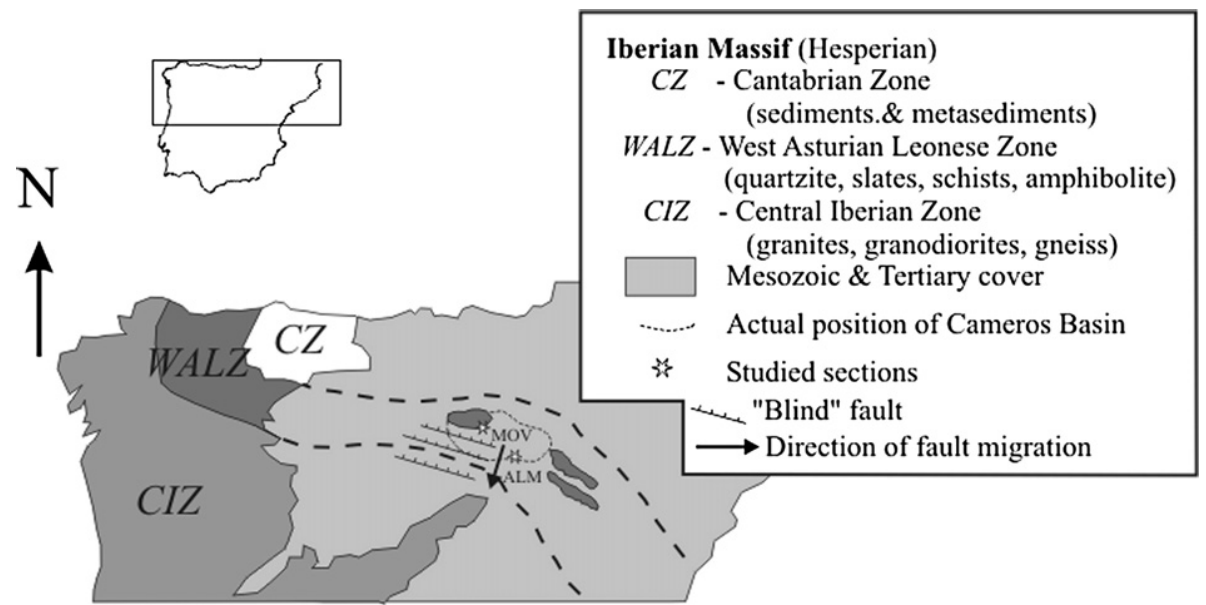

Fig. 6. Location of the West Asturian Leonese Zone (WALZ) and the Central Iberian Zone (CIZ) in the scheme of Julivert et al. (1972) for the Iberian (Hesperian) Massif. The situation of both sections is indicated as: MOV, Montenegro-Villoslada en Cameros (northwestern section) and ALM, Almajano (southeastern section). The arrow indicates the tectonic development of the western basin, from the late Jurassic to Middle Albian (modified from Arribas et al., 2003).

Medium sand-sized polycrystalline quartz grains derived from granitoid and gneissic sources are formed by quartz grains with few crystal units, typically less than three, whereas polycrystalline quartz grains derived from slate-schists tend to contain more than three crystal units per grain (Basu et al., 1975; Tortosa et al., 1991; Palomares and Arribas, 1993). The polycrystalline quartz at the top of both sections shows a tectonic fabric and is commonly

SE

NW

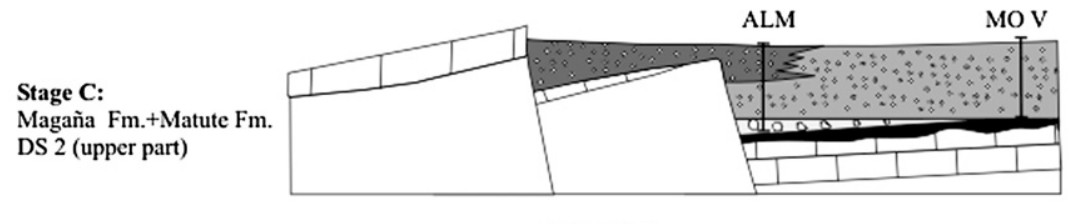

Stage B:

Magaña Fm.

DS 2 (lower part)

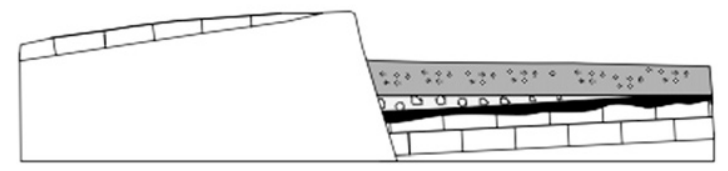

Stage A:

Ágreda Fm.

DS 1
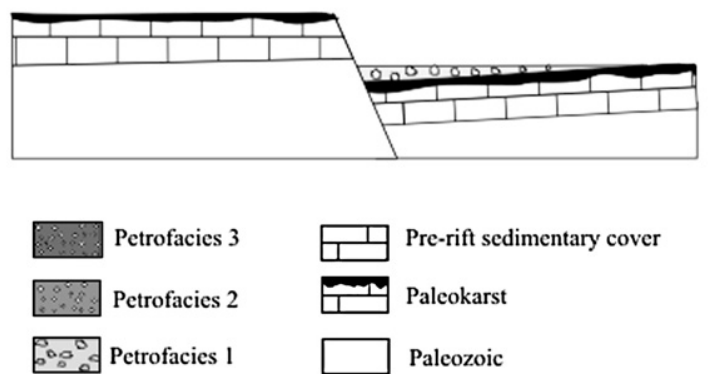

Fig. 7. Tectonic development of petrofacies during the Tithonian. Note that reactivation of the basin took place during deposition of Petrofacies 3 due to back faulting. The location of both sections is indicated as: MOV, Montenegro-Villoslada en Cameros (northwestern section) and ALM, Almajano (southeastern section). 
composed of more than three crystal units. Accordingly, its origin can be traced to metamorphic source areas.

In contrast, several factors point to plutonic sources for Petrofacies 2: (1) the scarcity of plutonic rock fragments in Petrofacies 2A and at the top of Petrofacies 2B. Granitoids have a very high potential to generate sands (Palomares and Arribas, 1993), but their mechanical isotropy produces sands formed by monomineral grains. Thus, plutonic sources generate scarce plutonic rock fragments; (2) high K-feldspar levels; and (3) the fact that Petrofacies 2 plots on the QmF edge of the QmFLt diagram (Fig. 5B).

The high muscovite content of Magaña Fm. 2 (see Fig. 3, Ms/Qmr ratio) also indicates an increase in the influence of both metamorphic and plutonic sources, due to the gradual unroofing of the basement.

The inferred sources for Petrofacies 2 (Magaña Fm., DS 2) are low-grade to medium-grade metamorphic terranes. These terranes probably correspond to the West Asturian Leonese Zone in the Iberian Massif (Fig. 6). However, the granite source rocks are probably from the Central Iberian Zone (Fig. 6). These interpretations are based on the main lithologies of both zones: the WestAsturian Leonese Zone consists mainly of a thick lower Paleozoic sequence of slates and quartzites of greenschist metamorphic facies and minor contents of amphibolite facies (Julivert, 1983). The main bedrock lithologies in the Central Iberian Zone are Hercynian granites, graniodiorites, and gneisses, with minor exposure of low-grade metamorphic rocks (Villaseca et al., 1993).

Moreover, different sources can be recognized in Petrofacies 2A and 2B. Establishing the provenance of Petrofacies 2B is more complex than that of Petrofacies 2A. Petrofacies 2B contains carbonate-rock fragments in Magaña Fm. 1 (Table 2 and Figs. 4E and 5). However, no carbonate-rock fragments were observed in Petrofacies $2 \mathrm{~A}$. Moreover, the $\mathrm{P} / \mathrm{K}$ ratio is higher for Petrofacies $2 \mathrm{~B}$ (5.14) than 2A (1.19). These observations suggest different source rocks for the two petrofacies, and probably indicate that the marine substrate was still being eroded during the deposition of the lower part of Petrofacies 2B.

An increase of K-feldspar content is recorded from Petrofacies 1 to Petrofacies 2, indicating an increasing depth of erosion. This tendency points to a change from undissected-transitional to transitional signatures (Garzanti et al., 2001, 2003). During the transitional stage, sands are derived from sedimentary successions and underlying basement rocks in varying proportions, as a function of the erosion level and type of rocks exposed (Garzanti et al., 2001, 2003).

Petrofacies 2 is equivalent to Petrofacies B (Arribas et al., 2003) from the western sector of the Cameros Basin.
The main difference between both petrofacies is the presence of plutonic rock fragments in Petrofacies 2, which are absent from Petrofacies B. Thus, deeper erosion of the basement was recorded in the eastern sector of the basin in sediments of Tithonian age.

Sedimentary sources are inferred for Petrofacies 3. In addition, a secondary influx of metamorphic sources is recorded. Sedimentary sources were probably generated by erosion of Jurassic marine sedimentary rocks, mainly Kimmeridgian limestones of the Torrecilla and Cameros Fm., as indicated by sparitic rock fragments and inherited echinoderm plates. This finding suggests reactivation of this part of the basin, probably due to back-faulting (Fig. 7), as has been inferred for the western part of the Cameros Basin (Arribas et al., 2003).

Petrofacies 3 is characteristic of the eastern sector of the Cameros Basin. This petrofacies is absent in the western sector. Moreover, Petrofacies 3 shows a signature between the undissected-transitional and the transitional stages of Garzanti et al. (2001, 2003), revealing the increasing relevance of sedimentary sources and the reactivation of this part of the basin.

Our provenance data broadly fit into the Garzanti et al. (2001, 2003) model for a modern rifted basin, which we consider a more appropriate conceptual framework than the more general Dickinsonian model (Dickinson et al., 1983). Furthermore, our study shows that the use of RgRsRm and LmLsmLse diagrams, and Qmr/Qmo, Qp/ $\mathrm{Qm}$ and $\mathrm{Ms} / \mathrm{Qmr}$ ratios are important for a comprehensive provenance analysis in rifted basins.

\section{Conclusions}

The present study of sandstone provenance in the initial rifting stage of the Cameros Basin illustrates that petrofacies analysis is a powerful tool for paleogeographic and paleotectonic reconstructions in tectonically controlled basins.

The Tera Group (Tithonian) records sediment accumulation during the initial formation of the Cameros Basin during the latest Jurassic-Early Cretaceous rift stage. The Tera Group is made up of alluvial-fan deposits, meandering fluvial sediments and lacustrine-palustrine carbonates.

Detrital modes of sandstones reveal three different petrofacies, which are closely related to lithostratigraphic units. As in other rifted basins, petrofacies indicate erosion of pre-rift sedimentary substratum at the beginning of the rifting, followed by unroofing of the basement in later stages. This progression was also recorded in the eastern part of Cameros Basin (Arribas et al., 2003) and in the modern Red Sea-Gulf of Aden system (Garzanti et al., 2001, 2003). 
Petrofacies 1 is sedimentolithic (mean: $\mathrm{Qm}_{54} \mathrm{~F}_{3} \mathrm{Lt}_{43}$ ) and developed during the deposition of the Ágreda Fm. (DS 1). This petrofacies mainly records erosion of the Jurassic marine sedimentary substrate.

The sandstones of Petrofacies 2 are quartzofeldspathic. Petrofacies 2 can be subdivided into Petrofacies 2A (mean: $\mathrm{Qm}_{84} \mathrm{~F}_{15} \mathrm{Lt}_{1}$ ) and Petrofacies 2B (mean: $\mathrm{Q}_{71} \mathrm{~F}_{23} \mathrm{Lt}_{6}$ ). This petrofacies records the sedimentation of the Magaña Fm. (DS 2). The deduced provenance terranes for the Magaña Fm. are low to medium grade metamorphics from the West Asturian Leonese Zone of the Iberian Massif, which represents the Paleozoic basement of the Cameros Basin. A minor influence of plutonic source areas from the Central Iberian Zone is also deduced. Progression from sedimentary sources to metamorphic sources from DS 1 to DS 2 is also observed in the western part of the Cameros Basin (Arribas et al., 2002). However, erosion of plutonic rocks (Petrofacies B in Arribas et al., 2003) has not been documented in the western part of the basin.

Petrofacies 3 (mean: $\mathrm{Qm}_{67} \mathrm{~F}_{16} \mathrm{Lt}_{17}$ ) characterizes the Sierra de Matute Fm. (DS 2). The sedimentary source for this formation is the marine Jurassic sedimentary substratum, as is the case for Petrofacies 1. This suggests an episode of reactivation of the basin, due to back-faulting. Thus, erosion of the pre-rift sedimentary substratum is followed by unroofing of the basement, as documented in other ancient and modern rifted basins.

\section{Acknowledgements}

Funding for this research was provided by the Spanish DIGICYT projects BTE 2001-026 and CGL 2005-07445C03-02/BTE. The authors would like to thank M.I. Benito, L. Domingo and A. Martín for their useful suggestions and comments. This manuscript also benefited from critical reviews by G.J. Weltje, C. Fielding and two anonymous referees.

\section{References}

Alonso, A., Mas, R., 1990. El Jurásico Superior marino en el sector de la Demanda-Cameros (La Rioja-Soria), vol. 14, pp. 173-198.

Alonso-Azcarate, J., Barrenechea, J.F., Rodas, M., Mas, R., 1995. Comparative study of the transition between very low grade metamorphism and low grade metamorphism in siliciclastic and carbonate sediments. Early Cretaceous, Cameros Basin (North Spain). Clay Minerals 30, 407-419.

Alonso-Azcarate, J., Rodas, M., Bottrell, S.H., Raiswell, R., Velasco, F., Mas, R., 1999. Pathways and distances of fluid flow during low grade metamorphism: evidence from pyrite deposits of the Cameros Basin, Spain. Journal of Metamorphic Geology 17 (4), 339-348.

Arribas, J., Tortosa, A., 2003. Detrital modes in sedimenticlastic sand from low-order streams in the Iberian Range, Spain: the potential for sand generation by different sedimentary rocks. Sedimentary Geology 159, 275-303.

Arribas, J., Gómez-Gras, D., Rosell, J., Tortosa, A., 1990. Estudio comparativo entre las areniscas Paleozoicas y Triásicas de la isla de Menorca: Evidencias de procesos de reciclado. Revista de la Sociedad Geológica de España 3, 105-116.

Arribas, A., Mas, R., Ochoa, M., Alonso, A., 2002. Composición y diagénesis del registro detrítico en el borde suroccidental de la cuenca de Cameros. Zubía. Instituto de Estudios Riojanos 14, 99-119.

Arribas, J., Alonso, A., Mas, R., Tortosa, A., Rodas, M., Barrenechea, J.F., Alonso-Azcarate, J., Artigas, R., 2003. Sandstone petrography of continental depositional sequences of a intraplate rift basin: Western Cameros Basin (North Spain). Journal of Sedimentary Research 73 (2), 309-327.

Basu, A., Young, S.W., Suttner, L.J., James, C.W., Mark, G.H., 1975. Re-evaluation of the use of undulatory extinction and polycrystallinity in detrital quartz provenance interpretation. Journal of Sedimentary Petrology 45, 873-882.

Barrenechea, F.J., Rodas, M., Frey, M., Alonso-Azcarate, J., Mas, J.R., 2000. Chlorite, Corrensite and Chlorite-Mica in late Jurassic Fluvio-Lacustrine sediments of the Cameros Basin of Northeastern Spain. Clay and Clay Minerals 48 (2), 256-265.

Barrenechea, F.J., Rodas, M., Frey, M., Alonso-Azcarate, J., Mas, J.R., 2001. Clay diagenesis and low-grade metamorphism of Tithonian and Berriasian sediments in the Cameros basin. Clay Minerals 36 (3), 325-333.

Benito, M.I., 2001. Estudio comparativo de la evolución sedimentaria y diagenética de los litosomas carbonatados arrecifales (pre-rifting) de la Cuenca de Cameros. Kimmeridgiense. La Rioja-Soria. Ph.D. Thesis. Universidad Complutense de Madrid, 410 pp.

Benito, M.I., Mas, R., 2001. Diagénesis temprana meteórica de la Fm. arrecifal Torrecilla en Cameros (Kimmeridgiense Inferior: prerift) y de los carbonatos de la base del Grupo Tera (Titónico: sinrift) en el sector de Soria. Cuenca de Cameros. N de España. Geotemas 3 (1), 83-88.

Benito, M.I., Mas, R., 2002. Evolución diagenética de los carbonatos arrecifales de la Formación Torrecilla en Cameros y de los carbonatos continentales suprayacentes (Kimmderidgiense inferior-Titónico) en el Sector de Soria. Cuenca de Cameros, N. de España. Journal of Iberian Geology 28, 65-92.

Benito, M.I., Lohmann, K.C., Mas, R., 2005. Late Jurassic palaeogeography and palaeoclimate in the Northern Iberian Basin of Spain: Constraints from diagenetic records in reefal and continental carbonates. Journal of Sedimentary Research 75, 82-96.

Blatt, H., 1967. Provenance determination and the recycling of sediments. Journal of Sedimentary Petrology 37, 1031-1044.

Casquet, C., Galindo, C., González-Casado, J.M., Alonso, A., Mas, R., Rodas, M., García, E., Barrenechea, J.F., 1992. El metamorfismo en la Cuenca de Los Cameros. Geocronología e implicaciones tectónicas. Geogaceta 11, 22-25.

Chayes, F., 1952. Notes of the staining of potash feldspar with sodium cobaltonitrite in thin section. American Mineralogist 37, 337-340.

Critelli, S., 1999. The interplay of lithosferic flexure and thrust accommodation in forming stratigraphic sequences in the southern Apennines foreland basin system. Italy: Lincei Scienze Fische e Naturali, Rendiconti, Series IX, 10, pp. 257-326.

Criteri, S., Le Pera, E., 1994. Detrital modes and provenance of Miocene sandstones and modern sands of the Southern Apennines thrust-top basins, Italy. Journal of Sedimentary Research, A 64, 824-835.

DeCelles, P.G., Hertel, F., 1989. Petrology of fluvial sands from the Amazonian foreland basin, Peru and Bolivia. Geological Society of America Bulletin 101, 1552-1562. 
Dickinson, W.R., 1970. Interpreting detrital modes of graywacke and arkose. Journal of Sedimentary Petrology 40, 695-707.

Dickinson, W.R., 1985. Interpreting provenance relations from detrital modes of sandstones. In: Zuffa, G.G. (Ed.), Provenance of Arenites. D. Reidel, Dordrecht, pp. 333-361.

Dickinson, W.R., Suczek, C.A., 1979. Plate tectonics and sandstone compositions. American Association of Petroleum Geologists 63, 2164-2182.

Dickinson, W.R., Beard, L.S., Barkenrigge, G.R., Erjavec, J.L., Ferguson, R.C., Inman, K.F., Knepp, R.A., Lindberg, F.A., Ryberg, P.T., 1983. Provenance of North America Phanerozoic sandstones in relation to tectonic setting. Geological Society of America Bulletin 94, 222-235.

Evans, A.L., 1990. Miocene sandstone provenance relations in the Gulf of Suez: insights into Synrift Unroofing and Uplift History. The American Association of Petroleum Geologists Bulletin 74, $1386-1400$.

Garzanti, E., Vezzoli, G., Andò, S., Castiglioni, G., 2001. Petrology of Rifted-Margin Sand (Red Sea and Gulf of Aden, Yemen). Journal of Geology 109, 277-297.

Garzanti, E., Andò, S., Vezzoli, G., Dell'Era, D., 2003. From rifted margins to foreland basins: investigating provenance and sediment dispersal across desert Arabia (Oman, U.A.E.). Journal of Sedimentary Research 73 (4), 572-588.

Gazzi, P., 1966. Le arenarie del flysch sopracretaceo dell'Appenino modenese; correlazioni con il flysch di Monghidoro. Mineralogica Petrografica Acta 12, 69-97.

Gómez Fernández, J.C., Meléndez, N., 1994. Estratigrafía de la Cuenca de los Cameros (Cordillera Ibérica Noroccidental, $\mathrm{N}$ de España) durante el tránsito Jurásico-Cretácico. Revista Sociedad Geológica de España 7 (1-2), 121-139.

Guimerà, J., Alonso, A., Mas, R., 1995. Inversion of an extensional-ramp basin by a newly formed thrust: the Cameros Basin (N Spain). In: Buchanan, J.G., Buchanan, P.G. (Eds.), Basin Inversion. Geological Society Special Publication, vol. 88, pp. 433-453.

Guiraud, M., Seguret, M., 1985. A realising solitary overstep model for the late Jurassic-Early Cretaceous (Wealdian) Soria strike-slip basin (Northern Spain). SEMP Special Publication 37, 159-175.

Ingersoll, R.V., 1978. Petrofacies and petrologic evolution of the Late Cretaceous forearc basin, northern and central California. Journal of Geology 86, 335-352.

Ingersoll, R.V., Bulard, T.F., Ford, R.L., Grimm, J.P., Pickle, J.D., Sares, S.W., 1984. The effect of grain size on detrital modes: a text of the Gazzi-Dickinson point-counting method. Journal of Sedimentary Petrology 54, 103-116.

Julivert, M., 1983. La estructura de la Zona Asturoccidental-Leonesa. In: Ríos, J.M. (Ed.), Geología de España. Libro Jubilar. Instituto Geológico y Minero de España, vol. T1, pp. 381-407.

Julivert, M., Fontboté, J.M, Ribeiro, A., Navais Conde, L.E., 1972. Mapa tectónico de la Península Ibérica y Baleares, E. 1:1.000.000. Instituto Geológico y Minero de España, Madrid.

Le Pera, E., Arribas, J., 2004. Sand composition in an Iberian passivemargin fluvial course: the Tajo River. Sedimentary Geology 171, 261-281.

Lindholm, R.C., Finkelman, R.B., 1972. Calcite staining: semiquantitative determination of ferrous iron. Journal of Sedimentary Petrology 42, 239-245.

Martín-Closas, M., Alonso Millán, A., 1998. Estratigrafía y Bioestratigrafía (Charophyta) del Cretácico inferior en el sector occidental de la Cuenca de Cameros (Cordillera Ibérica). Revista de la Sociedad Geológica de España 11, 253-269.

Mas, R., Alonso, A., Guimerà, J., 1993. Evolución tectonosedimentaria de una cuenca extensional intraplaca: la cuenca finijurásica- eocretácica de Los Cameros (La Rioja-Soria). Revista Sociedad Geológica de España 6 (3-4), 129-144.

Mas, R., Benito, M.I., Arribas, J., Serrano, A., Guimerà, J., Alonso, A., Alonso-Azcarate, J., 2002. La Cuenca de Cameros: desde la extensión finijurásica-eocretácica a la inversión terciaria implicaciones en la exploración de hidrocarburos. Zubía. Instituto de Estudios Riojanos 14, 9-64.

Mas, R., Benito, M.I., Arribas, J., Serrano, A., Alonso, A., AlonsoAzcarate, J., 2003. The Cameros Basin: From late Jurassic-Early Cretaceous Extension to Tertiary Contractional Inversion-Implications of Hydrocarbon Exploration. In: AAPG International Conference and Exhibition, Barcelona, Spain. Geological Field Trip 11 (52 pages).

Mas, R. (coord.), García, A (coord.), Salas, R., Meléndez, A., Alonso, A., Aurell, M., Bádenas, B., Benito, M.I., Carenas, B., GarcíaHidalgo, J.F., Gil, J., Segura, M., 2004. 5.3.3. Segunda fase de rifting: Jurásico Superior-Cretácico Inferior. In: Geología de España (J. Vera Ed.), Sociedad Geológica de España - Instituto Geológico y Minero de España, 503-509.

Palomares, M., Arribas, J., 1993. Modern stream sands from compound crystalline sources: composition and sand generation index. In: Johnsson, M.J., Basu, A. (Eds.), Processes Controlling the Composition of Clastic Sediments. Geological Society of America, Special Paper, vol. 284, pp. 313-322.

Pettijohn, F.J., Potter, P.E., Siever, R., 1972. Sand and Sandstones. Springer - Verlag, New York, p. 618.

Salas, R., Guimerá, J., Mas, R., Martín-Closas, C., Meléndez, A., Alonso, A., 2001. Evolution of the Mesozoic Central Iberian Rift System and its Cainozoic Inversion (Iberian Chain). In: Cavazza, W., Roberson, A.H.F.R., Ziegler, P. (Eds.), Peri-Tethyan RiftWrench Basins and Passive Margins. Mémoires du Muséum National d' Histoire Naturelle, vol. 186, pp. 145-185.

Schwab, F., 1986. Sedimentary "signatures" of foreland basin assemblages: real or counterfeit? In: Allen, P.A., Homewood, P. (Eds.), Foreland Basins: International Association of Sedimentologists. Special Publication, vol. 8, pp. 395-410.

Tortosa, A., Palomares, M., Arribas, J., 1991. Quartz grain types in Holocene deposits from the Spanish Central System: some problems in provenance analysis. Developments in Sedimentary Provenance Studies. Geological Society Special Publication, vol. 57 , pp. 47-54.

Valloni, R., 1985. Reading provenance from modern marine sands. In: Zuffa, G.G. (Ed.), Provenance of Arenites: The Netherlands, Dordrecht, Reidel, pp. 309-332.

Villaseca, C., Barbero, L., Huertas, M.J., Andonaegui, P., Bellido, F., 1993. A cross-section though Hercynian granites of the Central Iberian Zone, Excursion guide, Servicio de Publicaciones. Consejo Superior de Investigaciones Científicas, Madrid, p. 122.

Weltje, G.J., 2002. Quantitative analysis of detrital modes: statistically rigorous confidence regions in ternary diagrams and their use in sedimentary petrology. Earth-Science Reviews 57, 211-253.

Zuffa, G.G., 1980. Hybrid arenites: their composition and classification. Journal of Sedimentary Petrology 50, 21-29.

Zuffa, G.G., 1985. Optical analyses of arenites: influence of methodology on compositional results. In: Zuffa, G.G. (Ed.), Provenance of arenites: The Netherlands, Dordrecht, Reidel, pp. 165-189.

Zuffa, G.G., 1987. Unravelling hinterland and offshore palaeogeography from deep-water arenites. Marine Clastic Sedimentology, pp. 39-61. Chapter 2.

Zuffa, G.G., Gaudio, W., Rovito, S., 1980. Detrital mode evolution of the rifted continental-margin Longobucco sequence (Jurassic), Calabrian Arc, Italy. Journal of Sedimentary Petrology 50 (1), $51-61$. 